\title{
Factors Associated with Physical Activity and Sedentary Behavior in Older Adults from Six Low- and Middle-Income Countries
}

\author{
Cadeyrn J. Gaskin ${ }^{(1)}$ and Liliana Orellana * (1) \\ Biostatistics Unit, Faculty of Health, Deakin University, Geelong 3220, Australia; cadeyrn.gaskin@deakin.edu.au \\ * Correspondence: 1.orellana@deakin.edu.au; Tel.: +61-3-9251-7096
}

Received: 20 March 2018; Accepted: 27 April 2018; Published: 3 May 2018

\begin{abstract}
Rising life expectancy in low- and middle-income countries (LMIC), coupled with the increasing burden of non-communicable diseases, accentuates the importance of generating information to support public health strategies. With this aim in mind, the purpose of this study was to identify correlates of physical activity and sedentary behavior in LMIC. We analyzed Wave 1 data (collected 2007-2010) from the World Health Organization's longitudinal Study on global AGEing and adult health (SAGE), which focuses on nationally representative samples of adults aged 50 years and older from six countries (China, $n=13,157$; India, $n=6560$; Mexico, $n=2301$; Russian Federation, $n=3763$; South Africa, $n=3836$; and Ghana, $n=4305$ ). Associations of physical activity (operationalized as meeting physical activity guidelines of $\geq 150 \mathrm{~min}$ /week of moderate-to-vigorous physical activity or not) and sedentary behavior ( $\geq 4 \mathrm{~h}$ /day versus $<4 \mathrm{~h} /$ day) with demographic, health and health risk, functional, interpersonal, and environmental factors were assessed using multivariate logistic models. Across the six countries, we found fairly consistent and reasonably strong associations between both physical activity and sedentary behavior and several demographic factors (age and employment, in particular), self-reported health, instrumental activities of daily living, factors relating to socializing, and household location. Correlates of physical activity and sedentary behavior in LMIC appear to be similar to those found in high-income countries.
\end{abstract}

Keywords: physical activity; sedentary behavior; exercise; older adults; ageing; ecological framework; low- and middle-income countries

\section{Introduction}

Non-communicable disease age-standardized death rates in low- and middle-income countries (LMIC; 756 per 100,000 for men and 565 per 100,000 for women in 2008) exceed those of high-income countries (65\% and $85 \%$ higher for men and women, respectively) [1]. The negative effects of globalization, rapid unplanned urbanization, and increases in sedentary living mean that the burden of non-communicable diseases in these countries will probably grow at an increasingly faster pace [1]. These trends underpin the importance of global targets for the prevention and control of non-communicable diseases, such as a $10 \%$ relative reduction in the prevalence of insufficient physical activity [2]. Analysis of self-reported data from 51 mainly LMIC has shown that one-fifth of adults aged 50-59 (men: 17.1\%; women: $21.3 \%$ ) and a quarter of those aged 60-69 (men: $22.3 \%$; women: $28.6 \%$ ) could be classified as physically inactive [3]. Knowledge of the correlates of physical activity and sedentary behavior in older adults living in LMIC could assist with the implementation of initiatives to increase physical activity and reduce sedentary behavior in these countries.

Demographic and biological correlates of physical activity in LMIC (most studies have been conducted in Brazil and China) are consistent with those found in high-income countries [4]. In LMIC, 
males and younger people tend to be more active than females and older adults, respectively $[4,5]$. Evidence is equivocal on the relationship between wealth and physical activity, with some studies (mainly from Brazil and China) showing the wealthier are more active [4], whereas a recent study from Bangladesh showed that people regarding themselves as poor were more active than those classifying themselves as rich [5]. Those with greater social support are also likely to be physically active $[4,6]$. Environmental correlates of physical activity include urbanicity (those living in rural areas tend to be more active) [5,7], ownership of various household devices (inverse association with television, car, and computer ownership) $[8,9]$, and the built environment (e.g., density of bus stations and access to bike paths) [10]. Studies focusing on older adults in a broader number of LMIC are still needed.

Much less is known about the correlates of sedentary behavior in LMIC. In one Chinese study involving adults aged 30-79 with no history of major disease, sedentary leisure time was positively associated with body mass index (BMI) values, waist circumference, and body fat, after controlling for age, study area, education, and annual household income [11]. These findings are largely consistent with those found in (mainly) high-income countries [12]. Evidence is limited, however. More research is warranted to explore the correlates of sedentary behavior in LMIC.

With increasing life expectancy in LMIC [13], attention needs to be paid to the health and well-being of growing populations of older adults. A greater understanding of the correlates of physical activity and sedentary behavior in LMIC may assist in designing interventions for specific subpopulations in these countries. To this end, we investigated the correlates of physical activity and sedentary behavior in adults aged 50 and older from six LMIC (China, India, Mexico, Russian Federation, South Africa, and Ghana). Specifically, we assessed the extent to which physical activity and sedentary behavior were associated with demographic, health and health-risk, functional, interpersonal, and environmental factors.

\section{Materials and Methods}

\subsection{Design}

Wave 1 data from the World Health Organization's (WHO) longitudinal Study on global AGEing and adult health (SAGE) were used for this secondary cross-sectional analysis. SAGE uses multistage cluster sampling strategies in the collection of data from nationally-representative samples of adults aged 50 years and older from six countries (China, India, Mexico, Russian Federation, South Africa, and Ghana), as well as smaller, comparative samples of adults aged 18-49 [14]. The six countries provide a diverse representation of geographic regions, levels of economic development, and stages in demographic and health transitions, as well as including the two countries with the highest populations in the world. These six countries were classified as LMIC when data collection commenced in 2007 [15]. Details on the study methods have been published $[14,16]$. Briefly, each household was allocated to one of two mutually exclusive categories: (1) 50+ households and (2) 18-49 households. Face-to-face interviews were conducted from 2007 to 2010. For each household, one household questionnaire was completed, and all persons aged 50 and over were invited to participate in individual interviews. In all countries, a standardized instrument, methods, interviewer training, and translation protocols were used. The World Health Survey team led the translation of the instrument, based on WHO guidelines (refer to http:/ / www.who.int/substance_abuse/research_tools/translation/en/ [17]). This method involves forward translation, expert panel back-translation, pretesting and cognitive interviewing, and the development of a final version. SAGE received approval from the WHO Ethical Review Committee and the respective committees in each participating country. Written informed consent was obtained from all study participants. The SAGE dataset is publicly available upon request (http:/ / www.who.int/healthinfo/sage/en/ [18]).

For our analysis, data from the $50+$ households were used. The overall sample sizes were 13,157 for China, 6560 for India, 2301 for Mexico, 3763 for Russian Federation, 3836 for South Africa, and 4305 for Ghana. Response rates were high in China (92\% for adults aged 50-59 and 93\% for those 
aged 60 and older), India $(90 \%, 85 \%)$, Russian Federation (80\%, 84\%), South Africa $(76 \%, 80 \%)$, and Ghana $(76 \%, 80 \%)$, and lower in Mexico (42\%, 55\%) [14]. Potential correlates of physical activity or sedentary behavior, based on research literature or reason, were selected from the SAGE dataset. Consistent with previous work on the correlates of physical activity and sedentary behavior $[4,12,19]$, we used an ecological framework to group the factors. Such frameworks position the individual within an ecosystem, depicting interactions between factors proximal and distal to the individual. Here, we grouped factors as follows: demographic, health and health risk, functional, interpersonal, and environmental.

\subsection{Measures}

\subsubsection{Demographic Factors}

Self-reported factors were age (categorized as 50-59, 60-69, 70-79, and 80+ years), sex (male and female), education completed (never been to school, less than primary school, primary school, secondary/high school, and college or more), and employment (working, not working, and retired/too old to work). Household wealth (country-specific quintiles) was derived from household ownership of selected durable goods, dwelling characteristics, and access to services [20].

\subsubsection{Health and Health-Risk Factors}

BMI was computed from weight and height measurements and categorized as: underweight $\left(<18.5 \mathrm{~kg} / \mathrm{m}^{2}\right)$, normal weight $\left(18.5\right.$ to $\left.<25 \mathrm{~kg} / \mathrm{m}^{2}\right)$, overweight $\left(25\right.$ to $\left.<30 \mathrm{~kg} / \mathrm{m}^{2}\right)$, and obese $\left(\geq 30 \mathrm{~kg} / \mathrm{m}^{2}\right)$ [21-23]. Self-reported factors were alcohol use (never drunk; drunk in the past; one drink per day or less, on average; and more than one drink per day, on average), smoking and tobacco use (no, less than daily, and daily), number of non-communicable diseases (summed from self-reports of arthritis, stroke, angina, diabetes, chronic lung disease, asthma, hypertension, and cataracts), pain (Over the last 30 days how much of bodily aches or pains did you have? Response options: none, mild, moderate, severe, and extreme), and self-rated health (In general, how would you rate your health today? Response options: very good, good, moderate, bad, and very bad).

\subsubsection{Functional Factors}

Mobility/dismobility was assessed using a $4 \mathrm{~m}$ timed walk. Participants were instructed to walk at their normal walking pace over a flat, straight, obstacle-free surface. Dismobility was defined as a speed of $\leq 0.6 \mathrm{~m} / \mathrm{s}$ [24]. Visual acuity was measured using myopic and hyperopic tests with LogMAR charts (Tumbling " $E$ " Chart for $4 \mathrm{~m}$ testing and Tumbling " $\mathrm{E}$ " Near Vision Card for $40 \mathrm{~cm}$ testing. Precision Vision Ltd., 944 First Street, LaSalle, IL 61301, USA). Corrected vision for each eye was tested separately, with the result from the eye with the better vision used for the analysis. The results for distance vision $(4 \mathrm{~m})$ and near vision $(40 \mathrm{~cm})$ were categorized (mild or no impairment and moderate impairment or greater) using accepted criteria [25]. Verbal learning and memory were assessed using the WHO/UCLA Auditory Verbal Learning Test [26], for which various scoring options are possible [27]. Over three trials, participants were read the same list of 10 words and asked to recall as many words as they remembered. The mean number of recalled words over the three trials was used for the analysis (words recalled immediately). Participants were then involved in unrelated cognitive and physiological tests for approximately $10 \mathrm{~min}$, before being asked to recall the 10 words again (this time without the list of words being read to them). The difference between the mean number of recalled words over the first three trials and the number of recalled words following the delay was used for the analysis (words lost with delay). That is, if the mean number of words recalled for the first three trials was 9 and the number of words recalled following the delay was 7 , then the number of words lost with the delay is 2 (i.e., $9-7=2$ ). Difficulties with activities of daily living (ADL, 23 activities; e.g., sitting for long periods) and instrumental activities of daily living (IADL, 5 activities; e.g., taking care of household responsibilities) over the last 30 days were assessed using a 5-point Likert scale 
anchored with none and extreme/cannot do. The mean ADL and IADL scores were used for the analysis. Disability was measured using the screener version (12 items) of the World Health Organization Disability Assessment Schedule 2.0 (WHODAS 2.0) [28,29]. Simple scoring was used, because it is a more valid method than applying weights to the WHODAS 2.0 items [30]. Scores were transformed to a 100-point scale, where higher values indicate greater disability. Quality of life was assessed using the short, 8-item version of the WHO quality of life scales [31], with scores transformed to a 100-point scale, where higher values signify greater quality of life.

\subsubsection{Interpersonal Factors}

Emotional loneliness was measured as a single item (Did you feel lonely for much of the day yesterday? Response options: yes and no). Four questions focused on social participation-How often in the last 12 months have you: (1) had friends over to your home; (2) been in the home of someone who lives in a different neighborhood than you do or had them in your home; (3) socialized with coworkers outside of work; and (4) gotten out of the house/your dwelling to attend social meetings, activities, programs or events or to visit friends and relatives? The response options were never, once or twice per year, once or twice per month, and once or twice per week or more. Marital status was categorized as coupled (married or cohabitating) or uncoupled (separated, divorced, widowed, or never married).

\subsubsection{Environmental Factors}

Location was classified as urban (an area legally proclaimed as being urban, including towns, cities, and metropolitan areas) or rural (an area not classified as urban). Access to personalized motorized transport (defined as the possession of a car, motorbike, or both modes of transport by anyone in the household) and the presence of a computer in the household (response options: yes and no) were self-reported. Safety on the street after dark (How safe do you feel when walking down your street alone after dark?) and safety when at home (In general, how safe from crime and violence do you feel when you are alone at home?) were both self-reported using a 5-point Likert scale anchored with completely safe and not at all safe.

\subsubsection{Physical Activity and Sedentary Behavior}

The Global Physical Activity Questionnaire (GPAQ, version 2) was used to measure participation in physical activity and sedentary behavior [32]. The instrument assesses frequency (days in a typical week) and duration (hours and minutes in a typical day) of moderate and vigorous physical activity in each of three domains (work, travel, and recreation), as well as time spent in sedentary behaviors (sitting or reclining) on a typical day. Studies of concurrent validity have shown highly variable correlations between GPAQ scores (e.g., moderate-to-vigorous physical activity and sedentary behavior) and both accelerometer and pedometer data, but stronger associations with scores on the International Physical Activity Questionnaire [33,34]. Test-retest reliability has generally found to be adequate [33-35]. A binary variable indicating whether or not participants had met WHO physical activity guidelines (150 min/week or more of moderate-to-vigorous physical activity for adults aged 18-64 years and those aged 65 years and older [36]) was defined. Sedentary behavior was dichotomized as $\geq 4 \mathrm{~h} /$ day and $<4 \mathrm{~h} /$ day. Sitting for $\geq 4 \mathrm{~h} /$ day is associated with increased risk of all-cause mortality, except for those engaged in about 50-65 min or more of moderate intensity physical activity (or equivalent) per day [37].

\subsection{Analysis}

We conducted the analysis using SAS (version 9.4). For each country, we ran separate analyses for physical activity and sedentary behavior. Some of the factors were omitted from the analyses due to large amounts of missing or implausible data: education (South Africa), BMI (Mexico, Russian Federation, and South Africa), mobility (Russian Federation and South Africa), distance and near vision (Mexico and Russian Federation), number of words lost with delay (South Africa), and socializing with 
coworkers outside of work (South Africa). Multicollinearity was assessed among the variables in each set of factors (demographic, health and health risk, functional, interpersonal, and environmental factors) in a linear regression with time spent sedentary (in minutes) as the outcome. In each country, the scores for IADL, ADL, and disability were highly correlated (variance inflation factor $>4$ ). IADL (but neither ADL nor disability) was included in the adjusted models because IADL was more strongly related to both physical activity and sedentary behavior than the other two variables (ADL and disability). No further factors showed strong collinearity. Due to the large number of factors to be investigated, and following an ecological framework, we adopted a three-stage modeling approach as in Solomon et al. [38]. First, univariate logistic regressions were fitted with each independent factor as the sole covariate. Independent factors associated with the outcome variables $(p<0.10)$ were selected for the next phase of the analysis. The demographic factors were included in all multivariate models. Specifically, age, sex, and employment were always included, along with either or both of education and household wealth (both were retained if both $p<0.10$, otherwise the variable with the smaller $p$ value was retained). Second, we ran partially adjusted logistic regressions with each set of factors (e.g., health and health-risk factors) as predictors. Only those variables retained from the first stage of the analyses and the demographics factors were used in this second phase. Third, we performed fully adjusted logistic regressions with each of the factors retained from the second phase. The results for the unadjusted and fully adjusted regressions are reported. Analyses were run on the subsets of adults with complete data on the factors included in the fully adjusted models.

\section{Results}

\subsection{Physical Activity}

In the physical activity models, the sample sizes of adults with complete data on factors included in the models were 11,046 for China (84\% of SAGE participants aged 50 years and older), 5816 for India (89\%), 2045 for Mexico (89\%), 3441 for Russian Federation (91\%), 2975 for South Africa (86\%), and 3752 for Ghana $(87 \%)$. The percentages of adults meeting physical activity guidelines in each sample varied among the six countries: China (32\%), India (41\%), Mexico (27\%), Russian Federation (48\%), South Africa (21\%), and Ghana (59\%).

The descriptive statistics for adults meeting physical activity guidelines are provided in Table 1. The odds ratios estimated under unadjusted models including demographic, health and health risk, functional, interpersonal, and environmental factors are shown in Table S1. The estimates for the fully adjusted models are provided in Table 2 . The description of results focuses predominantly on the fully adjusted models.

\subsubsection{Demographic Factors}

Across the six countries, age, employment, and household wealth were the variables most consistently associated with meeting physical activity guidelines. The odds of meeting guidelines significantly decreased with age in all countries. Not working and being retired/too old to work were both significantly associated with lower odds of meeting guidelines in five countries (China, India, Mexico, South Africa, and Ghana), with the results for Russian Federation in the same direction. Adults from households in the lowest wealth quartiles were more likely to be meeting guidelines in China, India, South Africa, and Ghana. In Russian Federation, the reverse was found, however, whereby adults from lower income households had lower odds of meeting guidelines.

The relationship between sex and meeting physical activity guidelines was equivocal. Females were more likely to be meeting guidelines in India and Russian Federation, males were more likely to be meeting guidelines in Ghana, and no differences between the sexes were found for China, Mexico, and South Africa.

Education was related to meeting physical activity guidelines in China, India, and Ghana, but the directions of the associations were not consistent across countries. 
Table 1. Descriptive statistics for the samples of adults and for those meeting physical activity guidelines (active).

\begin{tabular}{|c|c|c|c|c|c|c|c|c|c|c|c|c|}
\hline \multirow{3}{*}{ Factors } & \multicolumn{2}{|c|}{ China } & \multicolumn{2}{|c|}{ India } & \multicolumn{2}{|c|}{ Mexico } & \multicolumn{2}{|c|}{ Russian Federation } & \multicolumn{2}{|c|}{ South Africa } & \multicolumn{2}{|c|}{ Ghana } \\
\hline & Sample & Active & Sample & Active & Sample & Active & Sample & Active & Sample & Active & Sample & Active \\
\hline & $n(\%)^{a}$ & $n(\%)^{b}$ & $\mathrm{n}(\%)^{\mathrm{a}}$ & $n(\%)^{b}$ & $n(\%)^{a}$ & $n(\%)^{b}$ & $n(\%)^{a}$ & $n(\%)^{b}$ & $n(\%)^{a}$ & $n(\%)^{b}$ & $n(\%)^{a}$ & $n(\%)^{b}$ \\
\hline \multicolumn{13}{|l|}{ Demographic Factors } \\
\hline \multicolumn{13}{|l|}{ Age } \\
\hline $50-59$ & $4941(45 \%)$ & $1951(40 \%)$ & $2716(47 \%)$ & $1401(52 \%)$ & $393(19 \%)$ & $143(36 \%)$ & $1303(38 \%)$ & $804(62 \%)$ & $1441(44 \%)$ & $425(30 \%)$ & $1508(40 \%)$ & $1037(69 \%)$ \\
\hline $60-69$ & $3320(30 \%)$ & $1061(32 \%)$ & $1983(34 \%)$ & $747(38 \%)$ & $845(41 \%)$ & $270(32 \%)$ & $940(27 \%)$ & $473(50 \%)$ & $1053(32 \%)$ & $173(16 \%)$ & $1054(28 \%)$ & $650(62 \%)$ \\
\hline 70-79 & $2248(20 \%)$ & $477(21 \%)$ & $887(15 \%)$ & $204(23 \%)$ & $564(28 \%)$ & $116(21 \%)$ & $871(25 \%)$ & $310(36 \%)$ & $593(18 \%)$ & $68(12 \%)$ & $836(22 \%)$ & $406(49 \%)$ \\
\hline $80+$ & $537(5 \%)$ & $57(11 \%)$ & $230(4 \%)$ & $26(11 \%)$ & $243(12 \%)$ & $21(9 \%)$ & $327(10 \%)$ & $61(19 \%)$ & $212(6 \%)$ & $19(9 \%)$ & $354(9 \%)$ & $136(38 \%)$ \\
\hline \multicolumn{13}{|l|}{ Sex } \\
\hline Male & $5109(46 \%)$ & $1820(36 \%)$ & $2965(51 \%)$ & $1267(43 \%)$ & $799(39 \%)$ & $260(33 \%)$ & $1231(36 \%)$ & $623(51 \%)$ & $1406(43 \%)$ & $347(25 \%)$ & $1968(53 \%)$ & $1277(65 \%)$ \\
\hline Female & $5937(54 \%)$ & $1726(29 \%)$ & $2851(49 \%)$ & $1111(39 \%)$ & $1246(61 \%)$ & $290(23 \%)$ & $2210(64 \%)$ & $1025(46 \%)$ & $1893(57 \%)$ & $338(18 \%)$ & $1784(48 \%)$ & $952(53 \%)$ \\
\hline \multicolumn{13}{|l|}{ Education completed ${ }^{c}$} \\
\hline Never been to school & $2672(24 \%)$ & $849(32 \%)$ & $2922(50 \%)$ & $1138(39 \%)$ & $408(20 \%)$ & $100(25 \%)$ & & & d & & & \\
\hline Less than primary school & $2047(19 \%)$ & $793(39 \%)$ & $647(11 \%)$ & $267(41 \%)$ & $850(42 \%)$ & $231(27 \%)$ & & & & & $2432(65 \%)$ & $1488(61 \%)$ \\
\hline Primary school & $2256(20 \%)$ & $845(38 \%)$ & $846(15 \%)$ & $381(45 \%)$ & $436(21 \%)$ & $126(29 \%)$ & $369(11 \%)$ & $103(28 \%)$ & & & $404(11 \%)$ & $241(60 \%)$ \\
\hline Secondary/high school & $3607(33 \%)$ & $1006(28 \%)$ & $1401(24 \%)$ & $592(42 \%)$ & $185(9 \%)$ & $60(32 \%)$ & $2397(70 \%)$ & $1192(50 \%)$ & & & $916(24 \%)$ & $500(55 \%)$ \\
\hline College or more & $464(4 \%)$ & $53(11 \%)$ & & & $166(8 \%)$ & $33(20 \%)$ & $675(20 \%)$ & $353(52 \%)$ & & & & \\
\hline \multicolumn{13}{|l|}{ Employment } \\
\hline Working & $2336(21 \%)$ & $592(25 \%)$ & $2573(44 \%)$ & $824(32 \%)$ & $1323(65 \%)$ & $271(21 \%)$ & $504(15 \%)$ & $217(43 \%)$ & $1320(40 \%)$ & $214(16 \%)$ & $657(18 \%)$ & $256(39 \%)$ \\
\hline Not working & $4431(40 \%)$ & $2119(48 \%)$ & $2484(43 \%)$ & $1413(57 \%)$ & $539(26 \%)$ & $258(48 \%)$ & $1141(33 \%)$ & $712(62 \%)$ & $869(26 \%)$ & $332(38 \%)$ & $2653(71 \%)$ & $1862(70 \%)$ \\
\hline Retired/too old to work & $4279(39 \%)$ & $835(20 \%)$ & $759(13 \%)$ & $141(19 \%)$ & $183(9 \%)$ & $21(12 \%)$ & $1796(52 \%)$ & $719(40 \%)$ & $1110(34 \%)$ & $139(13 \%)$ & $442(12 \%)$ & $111(25 \%)$ \\
\hline \multicolumn{13}{|l|}{ Household wealth } \\
\hline 1st (high) quintile & $2169(20 \%)$ & $776(36 \%)$ & $960(17 \%)$ & $462(48 \%)$ & $442(22 \%)$ & $126(29 \%)$ & $629(18 \%)$ & $226(36 \%)$ & $596(18 \%)$ & $153(26 \%)$ & $750(20 \%)$ & $491(66 \%)$ \\
\hline 2nd & $2231(20 \%)$ & $878(39 \%)$ & $1087(19 \%)$ & $509(47 \%)$ & $436(21 \%)$ & $104(24 \%)$ & $694(20 \%)$ & $286(41 \%)$ & $655(20 \%)$ & $151(23 \%)$ & $743(20 \%)$ & $469(63 \%)$ \\
\hline 3rd & $2236(20 \%)$ & $751(34 \%)$ & $1062(18 \%)$ & $449(42 \%)$ & $363(18 \%)$ & $98(27 \%)$ & $690(20 \%)$ & $329(48 \%)$ & $657(20 \%)$ & $128(20 \%)$ & $750(20 \%)$ & $500(67 \%)$ \\
\hline 4th & $2292(21 \%)$ & $702(31 \%)$ & $1252(22 \%)$ & $460(37 \%)$ & $417(20 \%)$ & $114(27 \%)$ & $690(20 \%)$ & $392(57 \%)$ & $689(21 \%)$ & $120(17 \%)$ & $773(21 \%)$ & $433(56 \%)$ \\
\hline 5th (low) quintile & $2118(19 \%)$ & $439(21 \%)$ & $1455(25 \%)$ & $498(34 \%)$ & $386(19 \%)$ & $108(28 \%)$ & $738(21 \%)$ & $415(56 \%)$ & $702(21 \%)$ & $133(19 \%)$ & $736(20 \%)$ & $336(46 \%)$ \\
\hline \multicolumn{13}{|c|}{ Health and Health-Risk Factors } \\
\hline \multicolumn{13}{|c|}{ Body mass index } \\
\hline Underweight & $506(5 \%)$ & $151(30 \%)$ & $2025(35 \%)$ & $814(40 \%)$ & d & & d & & $\mathrm{d}$ & & $581(16 \%)$ & $331(57 \%)$ \\
\hline Normal weight & $6896(62 \%)$ & $2382(35 \%)$ & $2953(51 \%)$ & $1251(42 \%)$ & & & & & & & $2126(57 \%)$ & $1368(64 \%)$ \\
\hline Overweight & $3060(28 \%)$ & $872(29 \%)$ & $666(12 \%)$ & $260(39 \%)$ & & & & & & & $700(19 \%)$ & $368(53 \%)$ \\
\hline Obese & $584(5 \%)$ & $141(24 \%)$ & $172(3 \%)$ & $53(31 \%)$ & & & & & & & $345(9 \%)$ & $162(47 \%)$ \\
\hline \multicolumn{13}{|l|}{ Alcohol use } \\
\hline Never drunk & $7664(69 \%)$ & $2152(28 \%)$ & $4885(84 \%)$ & $1950(40 \%)$ & $1066(52 \%)$ & $251(24 \%)$ & $915(27 \%)$ & $345(38 \%)$ & $2396(73 \%)$ & $490(21 \%)$ & $1535(41 \%)$ & $858(56 \%)$ \\
\hline Drunk in the past & $1274(12 \%)$ & $477(37 \%)$ & $551(10 \%)$ & $233(42 \%)$ & $755(37 \%)$ & $227(30 \%)$ & $1738(51 \%)$ & $835(48 \%)$ & $469(14 \%)$ & $113(24 \%)$ & $1176(31 \%)$ & $674(57 \%)$ \\
\hline$\leq 1$ drink per day & $849(8 \%)$ & $318(38 \%)$ & $293(5 \%)$ & $145(50 \%)$ & $182(9 \%)$ & $53(29 \%)$ & $626(18 \%)$ & $370(59 \%)$ & $252(8 \%)$ & $46(18 \%)$ & $564(15 \%)$ & $352(62 \%)$ \\
\hline$>1$ drink per day & $1259(11 \%)$ & $599(48 \%)$ & $87(2 \%)$ & $50(58 \%)$ & $42(2 \%)$ & $19(45 \%)$ & $162(5 \%)$ & $98(61 \%)$ & $179(5 \%)$ & $36(20 \%)$ & $477(13 \%)$ & $345(72 \%)$ \\
\hline \multicolumn{13}{|l|}{ Smoking and tobacco use } \\
\hline No & $8032(73 \%)$ & $2339(29 \%)$ & $3018(52 \%)$ & $1120(37 \%)$ & $1670(82 \%)$ & $443(27 \%)$ & $2828(82 \%)$ & $1311(46 \%)$ & $2429(74 \%)$ & $538(22 \%)$ & $3280(87 \%)$ & $1904(58 \%)$ \\
\hline Less than daily & $282(3 \%)$ & $97(34 \%)$ & $167(3 \%)$ & $48(29 \%)$ & $122(6 \%)$ & $35(29 \%)$ & $49(1 \%)$ & 18 (37\%) & $131(4 \%)$ & $14(11 \%)$ & $106(3 \%)$ & $76(72 \%)$ \\
\hline Daily & $2732(25 \%)$ & $1110(41 \%)$ & $2631(45 \%)$ & $1210(46 \%)$ & $253(12 \%)$ & $72(29 \%)$ & $559(16 \%)$ & $316(57 \%)$ & $739(22 \%)$ & $133(18 \%)$ & $366(10 \%)$ & $249(68 \%)$ \\
\hline
\end{tabular}


Table 1. Cont

\begin{tabular}{|c|c|c|c|c|c|c|c|c|c|c|c|c|}
\hline \multirow{3}{*}{ Factors } & \multicolumn{2}{|c|}{ China } & \multicolumn{2}{|c|}{ India } & \multicolumn{2}{|c|}{ Mexico } & \multicolumn{2}{|c|}{ Russian Federation } & \multicolumn{2}{|c|}{ South Africa } & \multicolumn{2}{|c|}{ Ghana } \\
\hline & Sample & Active & Sample & Active & Sample & Active & Sample & Active & Sample & Active & Sample & Active \\
\hline & $n(\%)^{a}$ & $n(\%)^{b}$ & $n(\%)^{a}$ & $n(\%)^{b}$ & $n(\%)^{a}$ & $n(\%)^{b}$ & $\mathrm{n}(\%)^{\mathrm{a}}$ & $n(\%)^{b}$ & $n(\%)^{a}$ & $n(\%)^{b}$ & $n(\%)^{a}$ & $n(\%)^{b}$ \\
\hline \multicolumn{13}{|c|}{ Health and Health-Risk Factors (Cont.) } \\
\hline \multicolumn{13}{|l|}{ Non-communicable diseases } \\
\hline 0 diseases & $5305(48 \%)$ & $2030(38 \%)$ & $3042(52 \%)$ & $1391(46 \%)$ & $776(38 \%)$ & $249(32 \%)$ & $825(24 \%)$ & $439(53 \%)$ & $1607(49 \%)$ & $414(26 \%)$ & $2526(67 \%)$ & $1609(64 \%)$ \\
\hline 1 disease & $3281(30 \%)$ & $1007(31 \%)$ & $1663(29 \%)$ & $656(39 \%)$ & $728(36 \%)$ & $187(26 \%)$ & $818(24 \%)$ & $450(55 \%)$ & $953(29 \%)$ & $187(20 \%)$ & $873(23 \%)$ & $472(54 \%)$ \\
\hline 2 diseases & $1585(14 \%)$ & $356(23 \%)$ & $733(13 \%)$ & $237(32 \%)$ & $368(18 \%)$ & $87(24 \%)$ & $805(23 \%)$ & $387(48 \%)$ & $465(14 \%)$ & $58(13 \%)$ & $271(7 \%)$ & $114(42 \%)$ \\
\hline $3+$ diseases & $875(8 \%)$ & $153(18 \%)$ & $378(7 \%)$ & $94(25 \%)$ & $173(9 \%)$ & $27(16 \%)$ & $993(29 \%)$ & $372(38 \%)$ & $274(8 \%)$ & $26(10 \%)$ & $82(2 \%)$ & $34(42 \%)$ \\
\hline \multicolumn{13}{|l|}{ Pain } \\
\hline None & $5773(52 \%)$ & $1792(31 \%)$ & $1585(27 \%)$ & $715(45 \%)$ & $866(42 \%)$ & $269(31 \%)$ & $1208(35 \%)$ & $650(54 \%)$ & $1205(37 \%)$ & $351(29 \%)$ & $725(19 \%)$ & $387(53 \%)$ \\
\hline Mild & $3632(33 \%)$ & $1233(34 \%)$ & $1971(34 \%)$ & $806(41 \%)$ & $566(28 \%)$ & $169(30 \%)$ & $996(29 \%)$ & $486(49 \%)$ & $966(29 \%)$ & $187(19 \%)$ & $1356(36 \%)$ & $929(69 \%)$ \\
\hline Moderate & $1342(12 \%)$ & $461(34 \%)$ & $1278(22 \%)$ & $500(39 \%)$ & $427(21 \%)$ & $83(19 \%)$ & $795(23 \%)$ & $354(45 \%)$ & $785(24 \%)$ & $101(13 \%)$ & $1108(30 \%)$ & $641(58 \%)$ \\
\hline Severe/extreme & $299(3 \%)$ & $60(20 \%)$ & $982(17 \%)$ & $357(36 \%)$ & $186(9 \%)$ & $29(16 \%)$ & $438(13 \%)$ & $156(36 \%)$ & $343(10 \%)$ & $46(13 \%)$ & $563(15 \%)$ & $272(48 \%)$ \\
\hline \multicolumn{13}{|l|}{ Self-rated health } \\
\hline Good/very good & $3755(34 \%)$ & $1359(36 \%)$ & $1685(29 \%)$ & $834(50 \%)$ & $760(37 \%)$ & $245(32 \%)$ & $442(13 \%)$ & $261(59 \%)$ & $1292(39 \%)$ & $401(31 \%)$ & $1530(41 \%)$ & $1044(68 \%)$ \\
\hline Moderate & $5047(46 \%)$ & $1527(30 \%)$ & $2990(51 \%)$ & $1188(40 \%)$ & $1024(50 \%)$ & $252(25 \%)$ & $2044(59 \%)$ & $1100(54 \%)$ & $1483(45 \%)$ & $228(15 \%)$ & $1616(43 \%)$ & $950(59 \%)$ \\
\hline Bad/very bad & $2244(20 \%)$ & $660(29 \%)$ & $1141(20 \%)$ & $356(31 \%)$ & $261(13 \%)$ & $53(20 \%)$ & $955(28 \%)$ & $287(30 \%)$ & $524(16 \%)$ & $56(11 \%)$ & $606(16 \%)$ & $235(39 \%)$ \\
\hline \multicolumn{13}{|l|}{ Functional Factors } \\
\hline \multicolumn{13}{|l|}{ Mobility } \\
\hline Mobility & $10451(95 \%)$ & $3468(33 \%)$ & $5087(88 \%)$ & $2191(43 \%)$ & $1502(73 \%)$ & $474(32 \%)$ & $\mathrm{d}$ & & d & & $2379(64 \%)$ & $1492(63 \%)$ \\
\hline Dismobility & $595(5 \%)$ & $78(13 \%)$ & $729(13 \%)$ & $187(26 \%)$ & $543(27 \%)$ & $76(14 \%)$ & & & & & $1315(36 \%)$ & $712(54 \%)$ \\
\hline \multicolumn{13}{|l|}{ Distance vision impairment } \\
\hline Mild or none & $9847(89 \%)$ & $3272(33 \%)$ & $4832(83 \%)$ & $2086(43 \%)$ & d & & d & & $2901(88 \%)$ & $632(22 \%)$ & $3296(88 \%)$ & $1971(60 \%)$ \\
\hline Moderate or greater & $1199(11 \%)$ & $274(23 \%)$ & $984(17 \%)$ & $292(30 \%)$ & & & & & $398(12 \%)$ & $53(13 \%)$ & $456(12 \%)$ & $258(57 \%)$ \\
\hline \multicolumn{13}{|l|}{ Near vision impairment } \\
\hline Mild or none & $7110(64 \%)$ & $2321(33 \%)$ & $3346(58 \%)$ & $1419(42 \%)$ & $\mathrm{d}$ & & $\mathrm{d}$ & & $2140(65 \%)$ & $455(21 \%)$ & $2730(73 \%)$ & $1665(61 \%)$ \\
\hline \multirow{2}{*}{ Moderate or greater } & $3936(36 \%)$ & $1225(31 \%)$ & $2458(42 \%)$ & $955(39 \%)$ & & & & & $1159(35 \%)$ & $230(20 \%)$ & $1021(27 \%)$ & $563(55 \%)$ \\
\hline & Mean (SD) & Mean (SD) & Mean (SD) & Mean (SD) & Mean (SD) & Mean (SD) & Mean (SD) & Mean (SD) & Mean (SD) & Mean (SD) & Mean (SD) & Mean (SD) \\
\hline \multicolumn{13}{|l|}{ Verbal learning and memory } \\
\hline Words recalled immediately $\mathrm{e}$ & $5.7(1.6)$ & $5.6(1.6)$ & $5.4(1.4)$ & $5.5(1.3)$ & $5.2(1.4)$ & $5.4(1.3)$ & $6.0(1.6)$ & $6.4(1.5)$ & $5.9(1.6)$ & $6.4(1.3)$ & $5.8(1.4)$ & $6.0(1.4)$ \\
\hline Words lost with delay ${ }^{\mathrm{e}}$ & $1.6(1.6)$ & $1.7(1.6)$ & $1.8(1.5)$ & $1.8(1.5)$ & $2.3(2.0)$ & $2.4(2.1)$ & $1.9(1.5)$ & $1.9(1.5)$ & d & & $1.8(1.6)$ & $1.8(1.7)$ \\
\hline $\mathrm{IADL}^{\mathrm{e}}$ & $0.21(0.48)$ & $0.11(0.29)$ & $0.94(0.86)$ & $0.77(0.73)$ & $0.65(0.89)$ & $0.32(0.51)$ & $0.66(0.88)$ & $0.38(0.57)$ & $0.54(0.84)$ & $0.21(0.50)$ & $0.72(0.79)$ & $0.58(0.61)$ \\
\hline $\mathrm{ADL}^{\mathrm{e}}$ & $0.23(0.40)$ & $0.15(0.26)$ & $0.87(0.69)$ & $0.71(0.58)$ & $0.76(0.76)$ & $0.47(0.49)$ & $0.68(0.73)$ & $0.45(0.51)$ & $0.62(0.72)$ & $0.34(0.49)$ & $0.73(0.67)$ & $0.61(0.54)$ \\
\hline Disability $^{\mathrm{e}}$ & $7.3(10.8)$ & $5.6(7.7)$ & $23.6(17.7)$ & $20.1(15.1)$ & $16.5(17.3)$ & $10.5(10.7)$ & $18.6(17.4)$ & $13.4(12.3)$ & $16.5(18.0)$ & $9.5(11.9)$ & $19.5(17.2)$ & $16.6(14.6)$ \\
\hline Quality of Life ${ }^{e}$ & $65.1(14.2)$ & $66.1(13.1)$ & $61.2(14.8)$ & $63.0(14.1)$ & $65.7(12.9)$ & $68.4(11.7)$ & $59.8(15.3)$ & $62.5(13.8)$ & $60.1(15.2)$ & $63.2(14.6)$ & $56.7(15.4)$ & $58.2(14.1)$ \\
\hline \multicolumn{13}{|l|}{ Interpersonal Factors } \\
\hline \multicolumn{13}{|l|}{ Emotional loneliness } \\
\hline No & $10364(94 \%)$ & $3361(32 \%)$ & $4883(84 \%)$ & $2030(42 \%)$ & $1681(82 \%)$ & $473(28 \%)$ & $2973(86 \%)$ & $1482(50 \%)$ & 2977 (91\%) & $629(21 \%)$ & $3370(90 \%)$ & $2050(61 \%)$ \\
\hline Yes & $623(6 \%)$ & $167(27 \%)$ & $933(16 \%)$ & $348(37 \%)$ & $364(18 \%)$ & $77(21 \%)$ & $468(14 \%)$ & $166(36 \%)$ & $310(9 \%)$ & $53(17 \%)$ & $382(10 \%)$ & $179(47 \%)$ \\
\hline
\end{tabular}


Table 1. Cont

\begin{tabular}{|c|c|c|c|c|c|c|c|c|c|c|c|c|}
\hline \multirow{3}{*}{ Factors } & \multicolumn{2}{|c|}{ China } & \multicolumn{2}{|c|}{ India } & \multicolumn{2}{|c|}{ Mexico } & \multicolumn{2}{|c|}{ Russian Federation } & \multicolumn{2}{|c|}{ South Africa } & \multicolumn{2}{|c|}{ Ghana } \\
\hline & Sample & Active & Sample & Active & Sample & Active & Sample & Active & Sample & Active & Sample & Active \\
\hline & $n(\%)^{a}$ & $n(\%)^{b}$ & $n(\%)^{a}$ & $n(\%)^{b}$ & $n(\%)^{a}$ & $n(\%)^{b}$ & $n(\%)^{a}$ & $n(\%)^{b}$ & $n(\%)^{a}$ & $n(\%)^{b}$ & $n(\%)^{a}$ & $n(\%)^{b}$ \\
\hline \multicolumn{13}{|l|}{ Interpersonal Factors (Cont.) } \\
\hline \multicolumn{13}{|l|}{ Friends visiting home } \\
\hline Never & $2935(27 \%)$ & $884(30 \%)$ & $1050(18 \%)$ & $396(38 \%)$ & $963(47 \%)$ & $216(22 \%)$ & $469(14 \%)$ & $161(34 \%)$ & $418(13 \%)$ & $53(13 \%)$ & $433(12 \%)$ & $149(34 \%)$ \\
\hline Once or twice per year & $4800(44 \%)$ & $1409(29 \%)$ & $1342(23 \%)$ & $514(38 \%)$ & $417(20 \%)$ & $117(28 \%)$ & $1408(41 \%)$ & $697(50 \%)$ & $410(12 \%)$ & $96(23 \%)$ & $365(10 \%)$ & $153(42 \%)$ \\
\hline Once or twice per month & $2481(23 \%)$ & $896(36 \%)$ & $1718(30 \%)$ & $728(42 \%)$ & $258(13 \%)$ & $78(30 \%)$ & $1192(35 \%)$ & $588(49 \%)$ & $979(30 \%)$ & $204(21 \%)$ & $774(21 \%)$ & $380(49 \%)$ \\
\hline Once per week or more & $830(8 \%)$ & $357(43 \%)$ & $1706(29 \%)$ & $740(43 \%)$ & $407(20 \%)$ & $139(34 \%)$ & $372(11 \%)$ & $202(54 \%)$ & $1492(45 \%)$ & $332(22 \%)$ & $2180(58 \%)$ & $1547(71 \%)$ \\
\hline \multicolumn{13}{|l|}{ Visiting people $^{\mathrm{f}}$} \\
\hline Never & $3902(35 \%)$ & $1101(28 \%)$ & $924(16 \%)$ & $333(36 \%)$ & $1063(52 \%)$ & $259(24 \%)$ & $1054(31 \%)$ & $404(38 \%)$ & $567(17 \%)$ & $74(13 \%)$ & $640(17 \%)$ & $221(35 \%)$ \\
\hline Once or twice per year & $4800(44 \%)$ & $1559(33 \%)$ & $1639(28 \%)$ & $666(41 \%)$ & $468(23 \%)$ & $142(30 \%)$ & $1504(44 \%)$ & $762(51 \%)$ & $608(18 \%)$ & $157(26 \%)$ & $506(14 \%)$ & $229(45 \%)$ \\
\hline Once or twice per month & $1598(15 \%)$ & $570(36 \%)$ & $1613(28 \%)$ & $653(41 \%)$ & $264(13 \%)$ & $80(30 \%)$ & $721(21 \%)$ & $387(54 \%)$ & $1085(33 \%)$ & $191(18 \%)$ & $935(25 \%)$ & $535(57 \%)$ \\
\hline Once per week or more & $746(7 \%)$ & $316(42 \%)$ & $1638(28 \%)$ & $726(44 \%)$ & $250(12 \%)$ & $69(28 \%)$ & $148(4 \%)$ & $86(58 \%)$ & $1039(32 \%)$ & $263(25 \%)$ & $1671(45 \%)$ & $1244(74 \%)$ \\
\hline \multicolumn{13}{|l|}{ Socializing with coworkers } \\
\hline Never & $3084(28 \%)$ & $850(28 \%)$ & $2380(41 \%)$ & $815(34 \%)$ & $1503(74 \%)$ & $352(23 \%)$ & $1672(49 \%)$ & $624(37 \%)$ & $\mathrm{d}$ & & $1551(41 \%)$ & $671(43 \%)$ \\
\hline Once or twice per year & $2773(25 \%)$ & $764(28 \%)$ & $1338(23 \%)$ & $527(39 \%)$ & $279(14 \%)$ & $94(34 \%)$ & $806(23 \%)$ & $441(55 \%)$ & & & $378(10 \%)$ & $203(54 \%)$ \\
\hline Once or twice per month & $2466(22 \%)$ & $764(31 \%)$ & $1045(18 \%)$ & $450(43 \%)$ & $139(7 \%)$ & $45(32 \%)$ & $552(16 \%)$ & $321(58 \%)$ & & & $575(15 \%)$ & $396(69 \%)$ \\
\hline Once per week or more & $2723(25 \%)$ & $1168(43 \%)$ & $1053(18 \%)$ & $586(56 \%)$ & $124(6 \%)$ & $59(48 \%)$ & $411(12 \%)$ & $262(64 \%)$ & & & $1248(33 \%)$ & $959(77 \%)$ \\
\hline \multicolumn{13}{|c|}{ Social activities outside of home } \\
\hline Never & $2080(19 \%)$ & $495(24 \%)$ & $1231(21 \%)$ & $392(32 \%)$ & $957(47 \%)$ & $223(23 \%)$ & $1260(37 \%)$ & $496(39 \%)$ & $774(24 \%)$ & $120(16 \%)$ & $483(13 \%)$ & $188(39 \%)$ \\
\hline Once or twice per year & $6567(60 \%)$ & $2201(34 \%)$ & $3215(55 \%)$ & $1355(42 \%)$ & $631(31 \%)$ & $196(31 \%)$ & $1002(29 \%)$ & $475(47 \%)$ & $741(23 \%)$ & $196(27 \%)$ & $647(17 \%)$ & $335(52 \%)$ \\
\hline Once or twice per month & $1956(18 \%)$ & $680(35 \%)$ & $1088(19 \%)$ & $496(46 \%)$ & $288(14 \%)$ & $81(28 \%)$ & $702(20 \%)$ & $387(55 \%)$ & $999(30 \%)$ & $233(23 \%)$ & $953(25 \%)$ & $559(59 \%)$ \\
\hline Once per week or more & $443(4 \%)$ & $170(38 \%)$ & $282(5 \%)$ & $135(48 \%)$ & $169(8 \%)$ & $50(30 \%)$ & $477(14 \%)$ & $290(61 \%)$ & $785(24 \%)$ & $136(17 \%)$ & $1669(45 \%)$ & $1147(69 \%)$ \\
\hline \multicolumn{13}{|l|}{ Marital status } \\
\hline Coupled & $9235(84 \%)$ & $3070(33 \%)$ & $4398(76 \%)$ & $1890(43 \%)$ & $1246(61 \%)$ & $385(31 \%)$ & $1948(57 \%)$ & $1037(53 \%)$ & $1750(53 \%)$ & $406(23 \%)$ & $2139(57 \%)$ & $1401(66 \%)$ \\
\hline Uncoupled & $1811(16 \%)$ & $476(26 \%)$ & $1418(24 \%)$ & $488(34 \%)$ & $799(39 \%)$ & $165(21 \%)$ & $1493(43 \%)$ & $611(41 \%)$ & $1549(47 \%)$ & $279(18 \%)$ & $1613(43 \%)$ & $828(51 \%)$ \\
\hline \multicolumn{13}{|l|}{ Environmental Factors } \\
\hline \multicolumn{13}{|l|}{ Location } \\
\hline Urban & $5230(47 \%)$ & $1135(22 \%)$ & $1444(25 \%)$ & $478(33 \%)$ & $1470(72 \%)$ & $378(26 \%)$ & $2609(76 \%)$ & $1190(46 \%)$ & $2212(67 \%)$ & $439(20 \%)$ & $1501(40 \%)$ & $685(46 \%)$ \\
\hline Rural & $5816(53 \%)$ & $2411(42 \%)$ & $4372(75 \%)$ & $1900(44 \%)$ & $575(28 \%)$ & $172(30 \%)$ & $832(24 \%)$ & $458(55 \%)$ & $1086(33 \%)$ & $246(23 \%)$ & $2251(60 \%)$ & $1544(69 \%)$ \\
\hline \multicolumn{13}{|l|}{ Personal motorized transport } \\
\hline No & $5014(45 \%)$ & $1735(35 \%)$ & $4291(74 \%)$ & $1859(43 \%)$ & $1300(64 \%)$ & $356(27 \%)$ & $1965(57 \%)$ & $845(43 \%)$ & $2428(74 \%)$ & $518(21 \%)$ & $3551(95 \%)$ & $2153(61 \%)$ \\
\hline Yes & $6032(55 \%)$ & $1811(30 \%)$ & $1525(26 \%)$ & $519(34 \%)$ & $744(36 \%)$ & $194(26 \%)$ & $1476(43 \%)$ & $803(54 \%)$ & $871(26 \%)$ & $167(19 \%)$ & $201(5 \%)$ & $76(38 \%)$ \\
\hline \multicolumn{13}{|l|}{ Computer in household } \\
\hline No & $8585(78 \%)$ & $3056(36 \%)$ & $5525(95 \%)$ & $2283(41 \%)$ & $1730(85 \%)$ & $478(28 \%)$ & $2782(81 \%)$ & $1294(47 \%)$ & $2827(86 \%)$ & $585(21 \%)$ & $3577(95 \%)$ & $2168(61 \%)$ \\
\hline Yes & $2461(22 \%)$ & $490(20 \%)$ & $291(5 \%)$ & $95(33 \%)$ & $314(15 \%)$ & $72(23 \%)$ & $659(19 \%)$ & $354(54 \%)$ & $455(14 \%)$ & $98(22 \%)$ & $175(5 \%)$ & $61(35 \%)$ \\
\hline \multicolumn{13}{|l|}{ Safety out on the street after $\mathrm{d}$. } \\
\hline Completely/very safe & $6635(60 \%)$ & $2340(35 \%)$ & $3549(61 \%)$ & $1521(43 \%)$ & $722(35 \%)$ & $199(28 \%)$ & $535(16 \%)$ & $261(49 \%)$ & $491(15 \%)$ & $119(24 \%)$ & $2751(73 \%)$ & $1696(62 \%)$ \\
\hline Moderately safe & $2872(26 \%)$ & $704(25 \%)$ & $1400(24 \%)$ & $523(37 \%)$ & $498(24 \%)$ & $133(27 \%)$ & $995(29 \%)$ & $497(50 \%)$ & $635(19 \%)$ & $136(21 \%)$ & $694(19 \%)$ & $405(58 \%)$ \\
\hline Slightly safe/not at all & $1539(14 \%)$ & $502(33 \%)$ & $867(15 \%)$ & $334(39 \%)$ & $825(40 \%)$ & $218(26 \%)$ & $1865(55 \%)$ & $875(47 \%)$ & $2149(66 \%)$ & $427(20 \%)$ & $307(8 \%)$ & $128(42 \%)$ \\
\hline
\end{tabular}


Table 1. Cont.

\begin{tabular}{|c|c|c|c|c|c|c|c|c|c|c|c|c|}
\hline \multirow{3}{*}{ Factors } & \multicolumn{2}{|c|}{ China } & \multicolumn{2}{|c|}{ India } & \multicolumn{2}{|c|}{ Mexico } & \multicolumn{2}{|c|}{ Russian Federation } & \multicolumn{2}{|c|}{ South Africa } & \multicolumn{2}{|c|}{ Ghana } \\
\hline & Sample & Active & Sample & Active & Sample & Active & Sample & Active & Sample & Active & Sample & Active \\
\hline & $n(\%)^{a}$ & $n(\%)^{b}$ & $n(\%)^{a}$ & $n(\%)^{b}$ & $n(\%)^{a}$ & $n(\%)^{b}$ & $n(\%)^{a}$ & $\mathrm{n}(\%)^{\mathrm{b}}$ & $n(\%)^{a}$ & $\mathrm{n}(\%)^{\mathrm{b}}$ & $\mathrm{n}(\%)^{\mathrm{a}}$ & $n(\%)^{b}$ \\
\hline \multicolumn{13}{|c|}{ Environmental Factors (Cont.) } \\
\hline $\begin{array}{l}\text { Safety when home alone } \\
\text { Completely/yerv safe }\end{array}$ & & $3012(32 \%)$ & $3924(68 \%)$ & $1668(43 \%)$ & $1133(55 \%)$ & $320(28 \%)$ & $1485(43 \%)$ & $740(50 \%)$ & $785(24 \%)$ & $214(27 \%)$ & $3186(85 \%)$ & $1996(63 \%)$ \\
\hline $\begin{array}{l}\text { Completely/very safe } \\
\text { Moderately safe }\end{array}$ & $\begin{array}{l}9283(84 \%) \\
1431(13 \%)\end{array}$ & $\begin{array}{l}4012(22 \%) \\
409(29 \%)\end{array}$ & $\begin{array}{l}3324(68 \%) \\
1364(24 \%)\end{array}$ & $\begin{array}{l}1608(43 \%) \\
519(38 \%)\end{array}$ & $\begin{array}{l}1133(53 \%) \\
422(21 \%)\end{array}$ & $\begin{array}{l}320(28 \%) \\
104(25 \%)\end{array}$ & $\begin{array}{l}14123(43 \%) \\
1123(33 \%)\end{array}$ & $\begin{array}{l}400(50 \%) \\
507(45 \%)\end{array}$ & $\begin{array}{l}785(24 \%) \\
904(27 \%)\end{array}$ & $\begin{array}{l}214(27 \%) \\
169(19 \%)\end{array}$ & $\begin{array}{l}3186(85 \%) \\
400(11 \%)\end{array}$ & $\begin{array}{l}1996(45 \%) \\
179(45 \%)\end{array}$ \\
\hline Slightly safe/not at all & $330(3 \%)$ & $123(37 \%)$ & $528(9 \%)$ & $191(36 \%)$ & $490(24 \%)$ & $126(26 \%)$ & $823(24 \%)$ & $393(48 \%)$ & $1610(49 \%)$ & $302(19 \%)$ & $166(4 \%)$ & $54(33 \%)$ \\
\hline
\end{tabular}

${ }^{a}$ Distribution of the factor in each country's sample. ${ }^{b}$ Percentage of people meeting physical activity guidelines (active) for each factor level. ${ }^{c}$ Education levels for some countries collapsed (e.g., secondary/high and college or more for India) due to low frequencies. ${ }^{\mathrm{d}}$ Factor omitted from the analyses due to large amounts of missing or implausible data. ${ }^{\mathrm{e}}$ Range: $0-10$ for words recalled immediately (higher scores = higher function) and words lost with delay (higher scores = lower function), $0-5$ for instrumental activities of daily living (IADL) and activities of daily living (ADL) (higher scores $=$ lower function), and 0-100 for disability (higher scores $=$ lower function) and quality of life (higher scores $=$ higher function). ${ }^{\mathrm{f}}$ Visiting people in different neighborhoods or them coming to visit.

Table 2. Multivariate associations of meeting physical activity guidelines with demographic, health and health risk, functional, interpersonal, and environmental factors.

\begin{tabular}{|c|c|c|c|c|c|c|c|c|c|c|c|c|}
\hline \multirow{2}{*}{ Factors } & \multicolumn{2}{|c|}{ China } & \multicolumn{2}{|c|}{ India } & \multicolumn{2}{|c|}{ Mexico } & \multicolumn{2}{|c|}{ Russian Federation } & \multicolumn{2}{|c|}{ South Africa } & \multicolumn{2}{|c|}{ Ghana } \\
\hline & OR $(95 \% \mathrm{CI})^{\mathrm{a}}$ & $p^{\mathrm{a}}$ & $\mathrm{OR}(95 \% \mathrm{CI})^{\mathrm{a}}$ & $p^{\mathrm{a}}$ & OR $(95 \% \mathrm{CI})^{\mathrm{a}}$ & $p^{\mathrm{a}}$ & OR $(95 \% \mathrm{CI})^{\mathrm{a}}$ & $p^{\mathrm{a}}$ & OR $(95 \% \mathrm{CI})^{\mathrm{a}}$ & $p^{\mathrm{a}}$ & OR $(95 \% \mathrm{CI})^{\mathrm{a}}$ & $p^{\mathrm{a}}$ \\
\hline \multicolumn{13}{|l|}{ Demographic Factors } \\
\hline \multicolumn{13}{|l|}{ Age (Ref: 50-59) } \\
\hline & $0.79(0.71,0.88)$ & $<0.0001$ & $0.76(0.67,0.87)$ & $<0.0001$ & $0.99(0.76,1.31)$ & 0.0347 & $0.74(0.60,0.90)$ & $<0.0001$ & $0.69(0.54,0.86)$ & 0.0008 & $0.79(0.64,0.97)$ & $<0.0001$ \\
\hline $70-79$ & $0.60(0.52,0.70)$ & & $0.50(0.41,0.61)$ & & $0.82(0.59,1.14)$ & & $0.54(0.42,0.68)$ & & $0.60(0.43,0.83)$ & & $0.50(0.40,0.64)$ & \\
\hline $80+$ & $0.38(0.27,0.52)$ & & $0.28(0.18,0.44)$ & & $0.48(0.27,0.82)$ & & $0.28(0.19,0.40)$ & & $0.49(0.28,0.82)$ & & $0.43(0.31,0.60)$ & \\
\hline \multicolumn{13}{|l|}{ Sex (Ref: Male) } \\
\hline Female & $0.97(0.86,1.10)$ & 0.6709 & $1.58(1.34,1.87)$ & $<0.0001$ & $1.13(0.86,1.50)$ & 0.3933 & $1.23(1.03,1.46)$ & 0.0199 & $0.83(0.68,1.02)$ & 0.0755 & $0.80(0.65,0.99)$ & 0.0408 \\
\hline \multicolumn{13}{|c|}{ Education completed (Ref: Lowest level, country-specific) ${ }^{b}$} \\
\hline Less than primary school & $1.02(0.89,1.17)$ & $<0.0001$ & $1.22(1.00,1.48)$ & 0.0003 & $0.97(0.72,1.30)$ & 0.1669 & & & c & & & \\
\hline Primary school & $1.08(0.94,1.25)$ & & $1.48(1.24,1.78)$ & & $0.89(0.63,1.26)$ & & & & & & $0.72(0.55,0.95)$ & $<0.0001$ \\
\hline College or more & $0.38(0.27,0.52)$ & & & & $0.54(0.33,0.89)$ & & $1.04(0.76,1.44)$ & & & & & \\
\hline \multicolumn{13}{|l|}{ Employment (Ref: Working) } \\
\hline Not working & $0.52(0.46,0.59)$ & $<0.0001$ & $0.45(0.39,0.52)$ & $<0.0001$ & & $<0.0001$ & & 0.4431 & & $<0.0001$ & & $<0.0001$ \\
\hline Retired/too old to work & $0.56(0.49,0.64)$ & & $0.29(0.23,0.36)$ & & $0.23(0.13,0.38)$ & & $0.91(0.73,1.13)$ & & $0.46(0.35,0.61)$ & & $0.32(0.24,0.42)$ & \\
\hline \multicolumn{13}{|c|}{ Household wealth (Ref: 1st (high) quintile) } \\
\hline 2nd & $1.32(1.12,1.56)$ & $<0.0001$ & $0.95(0.78,1.15)$ & $<0.0001$ & d & & $1.05(0.82,1.33)$ & 0.0007 & $1.27(0.94,1.71)$ & $<0.0001$ & $0.91(0.70,1.19)$ & 0.0055 \\
\hline $3 r d$ & $1.50(1.25,1.81)$ & & $1.19(0.95,1.48)$ & & & & $0.90(0.69,1.17)$ & & $1.41(1.04,1.91)$ & & $1.34(1.00,1.78)$ & \\
\hline 4th & $1.80(1.47,2.20)$ & & $1.51(1.20,1.90)$ & & & & $0.70(0.53,0.93)$ & & $1.71(1.27,2.32)$ & & $1.19(0.88,1.59)$ & \\
\hline 5th (low) quintile & $1.76(1.41,2.20)$ & & $1.67(1.31,2.13)$ & & & & $0.62(0.46,0.85)$ & & $2.23(1.64,3.04)$ & & $1.48(1.08,2.02)$ & \\
\hline
\end{tabular}


Table 2. Cont

\begin{tabular}{|c|c|c|c|c|c|c|c|c|c|c|c|c|}
\hline \multirow{2}{*}{ Factors } & \multicolumn{2}{|c|}{ China } & \multicolumn{2}{|c|}{ India } & \multicolumn{2}{|c|}{ Mexico } & \multicolumn{2}{|c|}{ Russian Federation } & \multicolumn{2}{|c|}{ South Africa } & \multicolumn{2}{|c|}{ Ghana } \\
\hline & OR $(95 \% \mathrm{CI})^{\mathrm{a}}$ & $p^{\text {a }}$ & OR $(95 \% \mathrm{CI})^{\text {a }}$ & $p^{\text {a }}$ & OR $(95 \% \mathrm{CI})^{\mathrm{a}}$ & $p^{\text {a }}$ & OR $(95 \% \mathrm{CI})^{\mathrm{a}}$ & $p^{\mathrm{a}}$ & OR $(95 \% \mathrm{CI})^{\mathrm{a}}$ & $p^{\mathrm{a}}$ & OR $(95 \% \mathrm{CI})^{\mathrm{a}}$ & $p^{\mathrm{a}}$ \\
\hline \multicolumn{13}{|c|}{ Health and Health-Risk Factors } \\
\hline \multicolumn{13}{|c|}{ Body mass index (Ref: Normal weight) } \\
\hline Underweight & $0.87(0.70,1.08)$ & 0.0060 & $0.94(0.82,1.07)$ & 0.3851 & c & & c & & c & & $0.95(0.75,1.20)$ & 0.0053 \\
\hline Overweight & $0.86(0.78,0.96)$ & & $1.04(0.86,1.26)$ & & & & & & & & $0.67(0.54,0.84)$ & \\
\hline Obese & $0.78(0.63,0.96)$ & & $0.79(0.55,1.12)$ & & & & & & & & $0.81(0.60,1.09)$ & \\
\hline \multicolumn{13}{|l|}{ Alcohol use (Ref: Never drunk) } \\
\hline Drunk in the past & $1.53(1.33,1.77)$ & $<0.0001$ & $1.07(0.86,1.31)$ & 0.8356 & $1.13(0.88,1.46)$ & 0.4463 & $1.46(1.22,1.75)$ & $<0.0001$ & d & & $1.18(0.97,1.44)$ & 0.3704 \\
\hline$\leq 1$ drink per day & $1.17(0.99,1.38)$ & & $1.11(0.85,1.45)$ & & $0.86(0.57,1.29)$ & & $1.76(1.39,2.23)$ & & & & $1.04(0.81,1.33)$ & \\
\hline$>1$ drink per day & $1.59(1.37,1.85)$ & & $1.08(0.68,1.74)$ & & $1.28(0.62,2.61)$ & & $1.83(1.25,2.68)$ & & & & $1.14(0.86,1.52)$ & \\
\hline \multicolumn{13}{|l|}{ Smoking and tobacco use (Ref: No) } \\
\hline Daily & $1.01(0.89,1.14)$ & & $1.22(1.07,1.38)$ & & & & & & $0.75(0.59,0.94)$ & & $1.07(0.79,1.44)$ & \\
\hline \multicolumn{13}{|c|}{ Non-communicable diseases (Ref: 0 diseases) } \\
\hline 1 disease & $0.87(0.78,0.96)$ & $<0.0001$ & $0.98(0.85,1.12)$ & 0.1466 & $0.91(0.71,1.16)$ & 0.7920 & $1.29(1.04,1.60)$ & 0.0723 & $0.93(0.75,1.16)$ & 0.1304 & $1.01(0.83,1.23)$ & 0.2363 \\
\hline 2 diseases & $0.73(0.63,0.84)$ & $=0.0001$ & $0.86(0.71,1.04)$ & 0.100 & $0.97(0.70,1.32)$ & 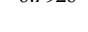 & $1.22(0.98,1.53)$ & . & $0.81(0.58,1.12)$ & . & $0.78(0.57,1.07)$ & . \\
\hline $3+$ diseases & $0.70(0.56,0.86)$ & & $0.77(0.58,1.01)$ & & $0.82(0.50,1.32)$ & & $1.08(0.86,1.37)$ & & $0.60(0.37,0.93)$ & & $1.41(0.80,2.46)$ & \\
\hline \multicolumn{13}{|l|}{ Pain (Ref: None) } \\
\hline Mild & $1.35(1.22,1.50)$ & $<0.0001$ & d & & $1.14(0.88,1.48)$ & 0.3482 & d & & $1.01(0.80,1.27)$ & 0.0171 & $2.53(2.00,3.20)$ & $<0.0001$ \\
\hline Moderate & $1.68(1.44,1.97)$ & & & & $0.86(0.61,1.19)$ & & & & $1.13(0.83,1.55)$ & & $2.12(1.64,2.75)$ & \\
\hline Severe/extreme & $1.10(0.79,1.53)$ & & & & $0.88(0.53,1.42)$ & & & & $2.00(1.27,3.12)$ & & $1.96(1.42,2.70)$ & \\
\hline \multicolumn{13}{|c|}{ Self-rated health (Ref: Good/very good) } \\
\hline Moderate & $0.88(0.79,0.98)$ & 0.0668 & $0.85(0.74,0.97)$ & 0.0398 & $0.83(0.66,1.06)$ & 0.1812 & $0.94(0.74,1.18)$ & $<0.0001$ & $0.63(0.50,0.80)$ & 0.0004 & $0.82(0.67,0.99)$ & $<0.0001$ \\
\hline Bad/very bad & $0.93(0.80,1.09)$ & & $0.81(0.67,0.99)$ & & $1.09(0.72,1.63)$ & & $0.53(0.40,0.71)$ & & $0.62(0.42,0.92)$ & & $0.49(0.38,0.65)$ & \\
\hline \multicolumn{13}{|l|}{ Mobility (Ref: Mobility) } \\
\hline Dismobility & $0.65(0.49,0.85)$ & 0.0018 & $0.81(0.66,0.99)$ & 0.0421 & $0.76(0.55,1.04)$ & 0.0851 & c & & c & & $1.22(0.95,1.58)$ & 0.1289 \\
\hline \multicolumn{13}{|c|}{ Distance vision impairment (Ref: Mild or none) } \\
\hline Moderate or greater & $0.83(0.71,0.98)$ & 0.0280 & $0.81(0.68,0.95)$ & 0.0110 & c & & c & & $0.81(0.58,1.12)$ & 0.2128 & d & \\
\hline \multicolumn{13}{|c|}{ Near vision impairment (Ref: Mild or none) } \\
\hline Moderate or greater & $1.03(0.94,1.14)$ & 0.5424 & d & & c & & c & & d & & d & \\
\hline \multicolumn{13}{|l|}{ Verbal learning and memory } \\
\hline Words recalled immediately $\mathrm{e}$ & d & & $1.05(1.00,1.10)$ & 0.0393 & d & & d & & $1.13(1.05,1.21)$ & 0.0005 & $1.15(1.08,1.23)$ & $<0.0001$ \\
\hline Words lost with delay $\mathrm{e}$ & d & & $\mathrm{d}$ & & $\mathrm{d}$ & & d & & $\mathrm{c}$ & & $1.01(0.96,1.06)$ & 0.8200 \\
\hline IADL $^{e}$ & $0.46(0.39,0.54)$ & $<0.0001$ & $0.85(0.78,0.92)$ & 0.0002 & $0.59(0.48,0.73)$ & $<0.0001$ & d & & $0.53(0.42,0.65)$ & $<0.0001$ & $0.79(0.69,0.91)$ & 0.0010 \\
\hline Quality of Life ${ }^{\mathrm{e}}$ & $1.00(1.00,1.01)$ & 0.5159 & d & & d & & d & & d & & d & \\
\hline \multicolumn{13}{|l|}{ Interpersonal Factors } \\
\hline \multicolumn{13}{|l|}{ Emotional loneliness (Ref: No) } \\
\hline Yes & $\mathrm{d}$ & & $0.98(0.83,1.16)$ & 0.8249 & $0.81(0.60,1.10)$ & 0.1796 & $0.99(0.79,1.26)$ & 0.9576 & d & & $1.18(0.90,1.54)$ & 0.2329 \\
\hline Friends visiting home (Ref: Neve & & & & & & & & & & & & \\
\hline Once or twice per year & $0.80(0.71,0.91)$ & 0.0005 & d & & $1.24(0.89,1.73)$ & 0.0074 & $1.21(0.95,1.54)$ & 0.0019 & $1.21(0.79,1.87)$ & 0.1728 & $0.84(0.57,1.22)$ & $<0.0001$ \\
\hline Once or twice per month & $0.91(0.79,1.05)$ & & & & $1.58(1.08,2.30)$ & & $0.86(0.67,1.12)$ & & $1.41(0.95,2.11)$ & & $1.10(0.79,1.54)$ & \\
\hline Once per week or more & $1.07(0.88,1.29)$ & & & & $1.78(1.26,2.52)$ & & $1.09(0.79,1.49)$ & & $1.56(1.04,2.35)$ & & $2.26(1.65,3.11)$ & \\
\hline
\end{tabular}


Table 2. Cont.

\begin{tabular}{|c|c|c|c|c|c|c|c|c|c|c|c|c|}
\hline \multirow{2}{*}{ Factors } & \multicolumn{2}{|c|}{ China } & \multicolumn{2}{|c|}{ India } & \multicolumn{2}{|c|}{ Mexico } & \multicolumn{2}{|c|}{ Russian Federation } & \multicolumn{2}{|c|}{ South Africa } & \multicolumn{2}{|c|}{ Ghana } \\
\hline & OR $(95 \% \mathrm{CI})^{\mathrm{a}}$ & $p^{\mathrm{a}}$ & OR $(95 \% \mathrm{CI})^{\mathrm{a}}$ & $p^{\mathrm{a}}$ & $\mathrm{OR}(95 \% \mathrm{CI})^{\mathrm{a}}$ & $p^{\mathrm{a}}$ & OR $(95 \% \mathrm{CI})^{\mathrm{a}}$ & $p^{\mathrm{a}}$ & OR $(95 \% \mathrm{CI})^{\mathrm{a}}$ & $p^{\mathrm{a}}$ & $\mathrm{OR}(95 \% \mathrm{CI})^{\mathrm{a}}$ & $p^{\mathrm{a}}$ \\
\hline \multicolumn{13}{|l|}{ Interpersonal Factors (Cont.) } \\
\hline \multicolumn{13}{|l|}{ Visiting people (Ref: Never) ${ }^{\mathrm{f}}$} \\
\hline Once or twice per year & $1.00(0.89,1.13)$ & 0.8431 & d & & $0.95(0.69,1.30)$ & 0.0187 & $1.39(1.15,1.69)$ & 0.0007 & $1.33(0.92,1.91)$ & 0.0001 & $1.25(0.91,1.72)$ & $<0.0001$ \\
\hline Once per week or more & $1.08(0.89,1.31)$ & & & & $0.53(0.35,0.80)$ & & $1.55(1.19,2.03)$ & & $1.15(0.79,1.68)$ & & $1.97(1.47,2.65)$ & \\
\hline \multicolumn{13}{|c|}{ Socializing with coworkers (Ref: Never) } \\
\hline Once or twice per year & $1.03(0.90,1.19)$ & $<0.0001$ & $1.02(0.87,1.19)$ & $<0.0001$ & $1.33(0.95,1.87)$ & 0.0514 & d & & c & & $1.31(0.98,1.75)$ & $<0.0001$ \\
\hline Once or twice per month & $1.02(0.88,1.17)$ & & $1.05(0.88,1.25)$ & & $1.00(0.65,1.53)$ & & & & & & $2.14(1.66,2.75)$ & \\
\hline Once per week or more & $1.32(1.15,1.51)$ & & $1.53(1.29,1.81)$ & & $1.72(1.10,2.68)$ & & & & & & $2.10(1.69,2.61)$ & \\
\hline \multicolumn{13}{|c|}{ Social activities outside of home (Ref: Never) } \\
\hline Once or twice per month & $1.52(1.30,1.78)$ & & $1.41(1.16,1.71)$ & & & & $1.37(1.11,1.69)$ & & $1.10(0.84,1.46)$ & & $1.23(0.91,1.67)$ & \\
\hline Once per week or more & $1.63(1.28,2.08)$ & & $1.27(0.95,1.70)$ & & & & $1.80(1.41,2.30)$ & & $0.78(0.57,1.07)$ & & $1.11(0.83,1.48)$ & \\
\hline \multicolumn{13}{|l|}{ Marital status (Ref: Coupled) } \\
\hline Uncoupled & $1.03(0.90,1.17)$ & 0.6973 & $1.02(0.88,1.18)$ & 0.8269 & $0.75(0.58,0.95)$ & 0.0195 & $0.94(0.80,1.12)$ & 0.5125 & $0.98(0.79,1.20)$ & 0.8305 & $0.80(0.65,0.98)$ & 0.0337 \\
\hline \multicolumn{13}{|l|}{ Environmental Factors } \\
\hline \multicolumn{13}{|l|}{ Location (Ref: Urban) } \\
\hline Rural & $1.16(1.01,1.32)$ & 0.0323 & $1.39(1.20,1.61)$ & $<0.0001$ & $1.17(0.91,1.50)$ & 0.2114 & $1.37(1.15,1.65)$ & 0.0006 & d & & $1.71(1.42,2.06)$ & $<0.0001$ \\
\hline \multicolumn{13}{|c|}{ Personal motorized transport (Ref: No) } \\
\hline Yes & $0.99(0.88,1.10)$ & 0.7874 & $0.81(0.68,0.96)$ & 0.0151 & d & & $0.98(0.82,1.18)$ & 0.8555 & $\mathrm{~d}$ & & $0.58(0.39,0.84)$ & 0.0046 \\
\hline \multicolumn{13}{|c|}{ Computer in household (Ref: No) } \\
\hline Yes & $0.75(0.64,0.88)$ & 0.0004 & d & & d & & $0.75(0.60,0.94)$ & 0.0121 & d & & $0.60(0.39,0.90)$ & 0.0149 \\
\hline Moderately safe & $0.78(0.70,0.86)$ & $<0.0001$ & d & & d & & d & & d & & $1.48(1.16,1.89)$ & 0.0004 \\
\hline Slightly safe/not at all & $1.20(1.05,1.37)$ & & & & & & & & & & $0.77(0.54,1.10)$ & \\
\hline \multicolumn{3}{|c|}{$\begin{array}{l}\text { Safety when home alone (Ref: Completely/very safe) } \\
\text { Moderately safe }\end{array}$} & & & & & & & & & & \\
\hline $\begin{array}{l}\text { Moderately safe } \\
\text { Slightly safe/not at all }\end{array}$ & d & & $\begin{array}{l}0.83(0.72,0.95) \\
0.77(0.62,0.94)\end{array}$ & 0.0033 & d & & d & & $\begin{array}{l}0.70(0.54,0.90) \\
0.68(0.54,0.85)\end{array}$ & 0.0022 & $0.56(0.41,0.76)$ & $<0.0001$ \\
\hline
\end{tabular}

${ }^{\mathrm{a}}$ Odds ratios (95\% confidence intervals) and $\mathrm{p}$ values for those factors that were selected in the fully adjusted final model for each country. ${ }^{\mathrm{b}}$ Education levels for some countries collapsed (e.g., secondary/high and college or more for India) due to low numbers. ${ }^{c}$ Factor omitted from the analyses due to large amounts of missing or implausible data. ${ }^{\mathrm{d}}$ Factor was not selected for the final model. ${ }^{\mathrm{e}}$ Higher scores $=$ higher function for words recalled immediately and quality of life; higher scores $=$ lower function for words lost with delay and IADL. ${ }^{\mathrm{f}}$ Visiting people in different neighborhoods or them coming to visit. 


\subsubsection{Health and Health-Risk Factors}

Poorer ratings of health were associated with being less likely to be meeting physical activity guidelines in India, Russian Federation, South Africa, and Ghana. In three countries (China, South Africa, and Ghana), people reporting pain had higher odds of meeting guidelines (these results contrast with the unadjusted estimates, where higher pain severity was associated with lower odds of meeting guidelines). Among the countries in which BMI was considered in the analysis (China, India, and Ghana), being overweight or obese (compared with normal weight) was related to lower odds of meeting physical activity guidelines in China and Ghana. Few significant relationships were found for meeting guidelines and alcohol use (China and Russian Federation), smoking and tobacco use (India and South Africa), and number of non-communicable diseases (China).

\subsubsection{Functional Factors}

Increased difficulties with IADL were associated with lower odds of meeting guidelines in all countries except Russian Federation (for which IADL was not selected for the final model). Other functional factors associated with meeting physical activity guidelines were mobility (China and India), distance vision (China and India), and verbal learning and memory (words recalled immediately; India, South Africa, and Ghana).

\subsubsection{Interpersonal Factors}

Factors measuring social connectedness were generally associated with increased odds of meeting physical activity guidelines. Adults who more frequently had friends visiting their homes (Mexico and Ghana), were visiting people in different neighborhoods or having them coming to visit (Russian Federation and Ghana), were socializing with coworkers outside of work (China, India, and Ghana), and were engaging in social activities outside of home (China, India, and Russian Federation) were more likely to be meeting guidelines. In contrast, adults visiting people in different neighborhoods or having them come to visit in Mexico were less likely to be meeting guidelines (this result was in the opposite direction to the corresponding univariate estimates, however). Adults who were coupled were more likely to be meeting guidelines (Mexico and Ghana). No significant relationships were found between meeting guidelines and emotional loneliness.

\subsubsection{Environmental Factors}

Meeting physical activity guidelines was more frequent among older adults living in rural areas in China, India, Russian Federation, and Ghana. Those who perceived themselves to be completely or very safe when home alone had higher odds of meeting guidelines than those who perceived themselves to be moderately safe or slightly safe/not at all safe (India, South Africa, and Ghana). Perceived safety out on the street after dark was associated with meeting guidelines in China and Ghana but the direction of the relationship was inconsistent. Adults reporting the presence of computers in their households were less likely to be meeting guidelines (China, Russian Federation, and Ghana). Meeting guidelines was associated with access to motorized transport only in India and Ghana.

\subsection{Sedentary Behavior}

For the sedentary behavior models, the sample sizes of adults with complete data on factors included in the models were 11,355 for China (86\% of SAGE participants aged 50 years and older), 6171 for India (94\%), 2052 for Mexico (89\%), 3446 for Russian Federation (92\%), 3033 for South Africa $(88 \%)$, and 3900 for Ghana (91\%). The percentages of adults sedentary for $\geq 4 \mathrm{~h} /$ day varied between the six samples: China (45\%), India (38\%), Mexico (21\%), Russian Federation (58\%), South Africa (37\%), and Ghana (43\%).

The descriptive statistics for adults sedentary for $\geq 4 \mathrm{~h}$ /day are provided in Table 3 . The odds ratios estimated under unadjusted models including demographic, health and health risk, functional, 
interpersonal, and environmental factors are shown in Table S2. The estimates for the fully adjusted models are provided in Table 4. The description of results focuses predominantly on the fully adjusted models.

\subsubsection{Demographic Factors}

Across countries, age and employment were the demographic factors most consistently associated with sedentary behavior. Increased age was related to higher odds of $\geq 4 \mathrm{~h} /$ day sedentary behavior in all countries except South Africa (although the association was in the same direction). Not working and being retired/too old to work were associated with higher odds of sedentary behavior compared with working (China, India, South Africa, and Ghana).

In some countries relationships were observed between $\geq 4 \mathrm{~h}$ /day sedentary behavior and sex (India and Mexico, where women were less likely to be sedentary), education (China, Mexico, and Russian Federation), and household wealth (China, India, and South Africa).

\subsubsection{Health and Health-Risk Factors}

Poorer self-rated health was associated with higher odds of $\geq 4 \mathrm{~h} /$ day sedentary behavior in China, Russian Federation, and Ghana (conversely, and in contrast to their respective univariate results, in India and South Africa, adults with poorer self-rated health had lower odds of $\geq 4 \mathrm{~h} /$ day sedentary behavior). Among the countries in which BMI was considered in the analysis (China, India, and Ghana), higher BMI was related to greater odds of $\geq 4 \mathrm{~h}$ /day sedentary behavior in China and Ghana. Few relationships were found between $\geq 4 \mathrm{~h}$ /day sedentary behavior and alcohol use (India, Russian Federation, and Ghana), smoking and tobacco use (India), and number of non-communicable diseases (China and India). No associations were present between sedentary behavior and pain.

\subsubsection{Functional Factors}

No functional factors were significantly related to $\geq 4 \mathrm{~h}$ /day sedentary behavior in more than three countries. Adults with dismobility had higher odds of $\geq 4 \mathrm{~h} /$ day sedentary behavior in China, India, and Mexico. Those with increased difficulties with IADL had higher odds of $\geq 4 \mathrm{~h}$ /day sedentary behavior in China, Mexico, and South Africa. Higher quality of life was associated with lower odds of $\geq 4 \mathrm{~h}$ /day sedentary behavior in India, South Africa, and Ghana.

Inconsistent relationships were present between $\geq 4 \mathrm{~h}$ /day sedentary behavior and verbal learning and memory factors (China, Mexico, and South Africa). Vision impairment was associated with higher odds of $\geq 4 \mathrm{~h} /$ day sedentary behavior in China (near vision) and South Africa and Ghana (distance vision).

\subsubsection{Interpersonal Factors}

People reporting emotional loneliness had higher odds of $\geq 4 \mathrm{~h} /$ day sedentary behavior in Mexico, Russian Federation, and Ghana. More frequent visits from friends was associated with increased odds of $\geq 4 \mathrm{~h}$ /day sedentary behavior for China and Ghana (a different association pattern was observed for South Africa, however, with more frequent visits related to lower odd of sedentary behavior). Increased frequency of visiting people in different neighborhoods or them coming to visit was related to lower odds of $\geq 4 \mathrm{~h} /$ day sedentary behavior for China, India, and Ghana. More frequent socializing with coworkers outside of work was associated with increased odds of $\geq 4 \mathrm{~h}$ /day sedentary behavior for India and Mexico, but lower odds for Russian Federation and Ghana. Increased frequency of social activities outside of home was related to higher odds of spending $\geq 4 \mathrm{~h} /$ day sedentary for China, South Africa, and Ghana. Being uncoupled was associated with slightly higher odds of $\geq 4 \mathrm{~h} /$ day sedentary behavior in China. 
Table 3. Descriptive statistics for the samples of adults and for those who were sedentary $\geq 4 \mathrm{~h} /$ day.

\begin{tabular}{|c|c|c|c|c|c|c|c|c|c|c|c|c|}
\hline \multirow{3}{*}{ Factors } & \multicolumn{2}{|c|}{ China } & \multicolumn{2}{|c|}{ India } & \multicolumn{2}{|c|}{ Mexico } & \multicolumn{2}{|c|}{ Russian Federation } & \multicolumn{2}{|c|}{ South Africa } & \multicolumn{2}{|c|}{ Ghana } \\
\hline & Sample & Sedentary & Sample & Sedentary & Sample & Sedentary & Sample & Sedentary & Sample & Sedentary & Sample & Sedentary \\
\hline & $n(\%)^{a}$ & $n(\%)^{b}$ & $n(\%)^{a}$ & $n(\%)^{b}$ & $n(\%)^{a}$ & $n(\%)^{b}$ & $n(\%)^{a}$ & $n(\%)^{b}$ & $n(\%)^{a}$ & $n(\%)^{b}$ & $n(\%)^{a}$ & $n(\%) b$ \\
\hline \multicolumn{13}{|l|}{ Demographic Factors } \\
\hline \multicolumn{13}{|l|}{ Age } \\
\hline $50-59$ & $5082(45 \%)$ & $1953(38 \%)$ & $2806(46 \%)$ & $888(32 \%)$ & $396(19 \%)$ & $58(15 \%)$ & $1317(38 \%)$ & $616(47 \%)$ & $1472(44 \%)$ & $467(32 \%)$ & $1561(40 \%)$ & $559(36 \%)$ \\
\hline $60-69$ & $3415(30 \%)$ & $1533(45 \%)$ & $2100(34 \%)$ & $800(38 \%)$ & $850(41 \%)$ & $148(17 \%)$ & $942(27 \%)$ & $552(59 \%)$ & $1087(32 \%)$ & $411(38 \%)$ & $1098(28 \%)$ & $449(41 \%)$ \\
\hline $70-79$ & $2306(20 \%)$ & $1242(54 \%)$ & $982(16 \%)$ & $476(49 \%)$ & $565(28 \%)$ & $133(24 \%)$ & $860(25 \%)$ & $559(65 \%)$ & $594(18 \%)$ & $265(45 \%)$ & $869(22 \%)$ & $449(52 \%)$ \\
\hline $80+$ & $552(5 \%)$ & $357(65 \%)$ & $283(5 \%)$ & $161(57 \%)$ & $241(12 \%)$ & $93(39 \%)$ & $327(10 \%)$ & $270(83 \%)$ & $209(6 \%)$ & $97(46 \%)$ & $372(10 \%)$ & $228(61 \%)$ \\
\hline \multicolumn{13}{|l|}{ Sex } \\
\hline Male & $5260(46 \%)$ & $2236(43 \%)$ & $3153(51 \%)$ & $1211(38 \%)$ & $798(39 \%)$ & $183(23 \%)$ & $1230(36 \%)$ & $690(56 \%)$ & $1438(43 \%)$ & $512(36 \%)$ & $2040(52 \%)$ & $789(39 \%)$ \\
\hline Female & $6095(54 \%)$ & $2849(47 \%)$ & $3018(49 \%)$ & $1114(37 \%)$ & $1254(61 \%)$ & $249(20 \%)$ & $2216(64 \%)$ & $1307(59 \%)$ & $1924(57 \%)$ & $728(38 \%)$ & $1860(48 \%)$ & $896(48 \%)$ \\
\hline \multicolumn{13}{|l|}{ Education completed ${ }^{c}$} \\
\hline Never been to school & $2766(24 \%)$ & $1193(43 \%)$ & 3144 (51\%) & $1160(37 \%)$ & $411(20 \%)$ & $87(21 \%)$ & & & d & & & \\
\hline Less than primary school & $2117(19 \%)$ & $845(40 \%)$ & $698(11 \%)$ & $267(38 \%)$ & $849(41 \%)$ & $177(21 \%)$ & & & & & $2526(65 \%)$ & $1134(45 \%)$ \\
\hline 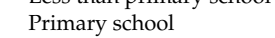 & $2320(20 \%)$ & $988(43 \%)$ & $881(14 \%)$ & $360(41 \%)$ & $438(21 \%)$ & $77(18 \%)$ & $367(11 \%)$ & $270(74 \%)$ & & & $419(11 \%)$ & $181(43 \%)$ \\
\hline Secondary/high school & $3672(32 \%)$ & $1763(48 \%)$ & $1448(24 \%)$ & $538(37 \%)$ & $186(9 \%)$ & $50(27 \%)$ & $2399(70 \%)$ & $1338(56 \%)$ & & & $955(25 \%)$ & $370(39 \%)$ \\
\hline College or more & $480(4 \%)$ & $296(62 \%)$ & & & $168(8 \%)$ & $41(24 \%)$ & $680(20 \%)$ & $389(57 \%)$ & & & & \\
\hline \multicolumn{13}{|l|}{ Employment } \\
\hline Working & $2396(21 \%)$ & $1149(48 \%)$ & $2753(45 \%)$ & $1182(43 \%)$ & $1329(65 \%)$ & $279(21 \%)$ & $493(14 \%)$ & $311(63 \%)$ & $1363(41 \%)$ & $553(41 \%)$ & $676(17 \%)$ & $400(59 \%)$ \\
\hline Not working & $4583(40 \%)$ & $1547(34 \%)$ & $2583(42 \%)$ & $726(28 \%)$ & $543(27 \%)$ & $93(17 \%)$ & $1158(34 \%)$ & $531(46 \%)$ & $887(26 \%)$ & $223(25 \%)$ & $2770(71 \%)$ & $983(36 \%)$ \\
\hline Retired/too old to work & $4376(39 \%)$ & $2389(55 \%)$ & $835(14 \%)$ & $417(50 \%)$ & $180(9 \%)$ & $60(33 \%)$ & $1795(52 \%)$ & $1155(64 \%)$ & $1112(33 \%)$ & $464(42 \%)$ & $454(12 \%)$ & $302(67 \%)$ \\
\hline \multicolumn{13}{|l|}{ Household wealth } \\
\hline 1st (high) quintile & $2229(20 \%)$ & $821(37 \%)$ & $1016(17 \%)$ & $403(40 \%)$ & $442(22 \%)$ & $83(19 \%)$ & $624(18 \%)$ & $401(64 \%)$ & $614(18 \%)$ & $247(40 \%)$ & $781(20 \%)$ & $351(45 \%)$ \\
\hline 2nd & $2295(20 \%)$ & $896(39 \%)$ & $1157(19 \%)$ & $469(41 \%)$ & $439(21 \%)$ & $92(21 \%)$ & $694(20 \%)$ & $431(62 \%)$ & $663(20 \%)$ & $294(44 \%)$ & $760(20 \%)$ & $330(43 \%)$ \\
\hline $3 \mathrm{rd}$ & $2302(20 \%)$ & $1018(44 \%)$ & $1141(19 \%)$ & $427(37 \%)$ & $365(18 \%)$ & $80(22 \%)$ & $689(20 \%)$ & $394(57 \%)$ & $661(20 \%)$ & $240(36 \%)$ & $775(20 \%)$ & $301(39 \%)$ \\
\hline 4 th & $2345(21 \%)$ & $1185(51 \%)$ & $1330(22 \%)$ & $437(33 \%)$ & $418(20 \%)$ & $99(24 \%)$ & $694(20 \%)$ & $378(55 \%)$ & $705(21 \%)$ & $235(33 \%)$ & $799(21 \%)$ & $347(43 \%)$ \\
\hline 5th (low) quintile & $2184(19 \%)$ & $1165(53 \%)$ & $1527(25 \%)$ & $589(39 \%)$ & $387(19 \%)$ & $77(20 \%)$ & $745(22 \%)$ & $393(53 \%)$ & $719(21 \%)$ & $224(31 \%)$ & $785(20 \%)$ & $356(45 \%)$ \\
\hline \multicolumn{13}{|c|}{ Health and Health-Risk Factors } \\
\hline \multicolumn{13}{|c|}{ Body mass index } \\
\hline $\begin{array}{l}\text { Underweight } \\
\text { Und }\end{array}$ & $515(5 \%)$ & $231(45 \%)$ & $2181(35 \%)$ & $893(41 \%)$ & $\mathrm{d}$ & & d & & d & & $600(15 \%)$ & $276(46 \%)$ \\
\hline Normal weight & $7091(62 \%)$ & $2971(42 \%)$ & $3112(50 \%)$ & $1129(36 \%)$ & & & & & & & $2204(57 \%)$ & $894(41 \%)$ \\
\hline Overweight & $3156(28 \%)$ & $1564(50 \%)$ & $696(11 \%)$ & $239(34 \%)$ & & & & & & & $734(19 \%)$ & $333(45 \%)$ \\
\hline Obese & $593(5 \%)$ & $319(54 \%)$ & $182(3 \%)$ & $64(35 \%)$ & & & & & & & $362(9 \%)$ & $182(50 \%)$ \\
\hline \multicolumn{13}{|l|}{ Alcohol use } \\
\hline Never drunk & $7867(69 \%)$ & $3657(47 \%)$ & $5177(84 \%)$ & $1956(38 \%)$ & $1069(52 \%)$ & $214(20 \%)$ & $914(27 \%)$ & $497(54 \%)$ & $2433(73 \%)$ & $883(36 \%)$ & $1598(41 \%)$ & $684(43 \%)$ \\
\hline Drunk in the past & $1301(12 \%)$ & $590(45 \%)$ & $592(10 \%)$ & $265(45 \%)$ & $758(37 \%)$ & $178(24 \%)$ & $1742(51 \%)$ & $1070(61 \%)$ & $480(14 \%)$ & $175(37 \%)$ & $1223(31 \%)$ & $597(49 \%)$ \\
\hline$\leq 1$ drink per day & $868(8 \%)$ & $327(38 \%)$ & $311(5 \%)$ & $69(22 \%)$ & $183(9 \%)$ & $35(19 \%)$ & $627(18 \%)$ & $333(53 \%)$ & $257(8 \%)$ & $93(36 \%)$ & $586(15 \%)$ & $227(39 \%)$ \\
\hline$>1$ drink per day & $1319(12 \%)$ & $511(39 \%)$ & $91(2 \%)$ & $35(39 \%)$ & $42(2 \%)$ & $5(12 \%)$ & $163(5 \%)$ & $97(60 \%)$ & $188(6 \%)$ & $88(47 \%)$ & $493(13 \%)$ & $177(36 \%)$ \\
\hline \multicolumn{13}{|l|}{ Smoking and tobacco use } \\
\hline No & $8225(73 \%)$ & $3817(46 \%)$ & $3199(52 \%)$ & $1179(37 \%)$ & $1676(82 \%)$ & $346(21 \%)$ & $2833(82 \%)$ & $1665(59 \%)$ & $2461(74 \%)$ & $913(37 \%)$ & $3397(87 \%)$ & $1498(44 \%)$ \\
\hline Less than daily & $287(3 \%)$ & $106(37 \%)$ & $187(3 \%)$ & $65(35 \%)$ & $123(6 \%)$ & $27(22 \%)$ & $48(1 \%)$ & $28(58 \%)$ & $130(4 \%)$ & $41(32 \%)$ & $112(3 \%)$ & $37(33 \%)$ \\
\hline Daily & $2811(25 \%)$ & $1153(41 \%)$ & $2785(45 \%)$ & $1081(39 \%)$ & $253(12 \%)$ & $59(23 \%)$ & $560(16 \%)$ & $300(54 \%)$ & $749(22 \%)$ & $280(37 \%)$ & $382(10 \%)$ & $146(38 \%)$ \\
\hline
\end{tabular}


Table 3. Cont

\begin{tabular}{|c|c|c|c|c|c|c|c|c|c|c|c|c|}
\hline \multirow{3}{*}{ Factors } & \multicolumn{2}{|c|}{ China } & \multicolumn{2}{|c|}{ India } & \multicolumn{2}{|c|}{ Mexico } & \multicolumn{2}{|c|}{ Russian Federation } & \multicolumn{2}{|c|}{ South Africa } & \multicolumn{2}{|c|}{ Ghana } \\
\hline & Sample & Sedentary & Sample & Sedentary & Sample & Sedentary & Sample & Sedentary & Sample & Sedentary & Sample & Sedentary \\
\hline & $n(\%)^{a}$ & $n(\%)^{b}$ & $n(\%)^{a}$ & $n(\%)^{b}$ & $n(\%)^{a}$ & $n(\%)^{b}$ & $n(\%)^{a}$ & $n(\%)^{b}$ & $n(\%)^{a}$ & $n(\%)^{b}$ & $n(\%)^{a}$ & $n(\%)^{b}$ \\
\hline \multicolumn{13}{|l|}{$\begin{array}{l}\text { Health and Health-Risk Factors } \\
\text { (Cont.) }\end{array}$} \\
\hline \multicolumn{13}{|l|}{ Non-communicable diseases } \\
\hline 0 diseases & $5446(48 \%)$ & $2107(39 \%)$ & $3159(51 \%)$ & $1085(34 \%)$ & $779(38 \%)$ & $151(19 \%)$ & $833(24 \%)$ & $395(47 \%)$ & $1644(49 \%)$ & $585(36 \%)$ & $2616(67 \%)$ & $1047(40 \%)$ \\
\hline 1 disease & $3366(30 \%)$ & $1567(47 \%)$ & $1772(29 \%)$ & $731(41 \%)$ & $729(36 \%)$ & $149(20 \%)$ & $818(24 \%)$ & $428(52 \%)$ & $967(29 \%)$ & $375(39 \%)$ & $916(24 \%)$ & $424(46 \%)$ \\
\hline 2 diseases & $1646(15 \%)$ & $893(54 \%)$ & $816(13 \%)$ & $331(41 \%)$ & $369(18 \%)$ & $83(23 \%)$ & $811(24 \%)$ & $483(60 \%)$ & $473(14 \%)$ & $184(39 \%)$ & $284(7 \%)$ & $157(55 \%)$ \\
\hline $3+$ diseases & $897(8 \%)$ & $518(58 \%)$ & $424(7 \%)$ & $178(42 \%)$ & $175(9 \%)$ & $49(28 \%)$ & $984(29 \%)$ & $691(70 \%)$ & $278(8 \%)$ & $96(35 \%)$ & $84(2 \%)$ & $57(68 \%)$ \\
\hline \multicolumn{13}{|l|}{ Pain } \\
\hline None & $5917(52 \%)$ & $2550(43 \%)$ & $1650(27 \%)$ & $566(34 \%)$ & $874(43 \%)$ & $159(18 \%)$ & $1223(36 \%)$ & $590(48 \%)$ & $1232(37 \%)$ & $388(32 \%)$ & $769(20 \%)$ & $267(35 \%)$ \\
\hline Mild & $3744(33 \%)$ & $1683(45 \%)$ & $2080(34 \%)$ & $726(35 \%)$ & $567(28 \%)$ & $105(19 \%)$ & $1002(29 \%)$ & $586(59 \%)$ & $966(29 \%)$ & $365(38 \%)$ & $1408(36 \%)$ & $574(41 \%)$ \\
\hline Moderate & $1382(12 \%)$ & $679(49 \%)$ & $1380(22 \%)$ & $545(40 \%)$ & $427(21 \%)$ & $107(25 \%)$ & $795(23 \%)$ & $525(66 \%)$ & $802(24 \%)$ & $329(41 \%)$ & $1132(29 \%)$ & $525(46 \%)$ \\
\hline Severe/extreme & $312(3 \%)$ & $173(55 \%)$ & $1061(17 \%)$ & $488(46 \%)$ & $184(9 \%)$ & $61(33 \%)$ & $426(12 \%)$ & $296(70 \%)$ & $359(11 \%)$ & $158(44 \%)$ & $588(15 \%)$ & $317(54 \%)$ \\
\hline \multicolumn{13}{|l|}{ Self-rated health } \\
\hline Good/very good & $3851(34 \%)$ & $1575(41 \%)$ & $1758(29 \%)$ & $608(35 \%)$ & $763(37 \%)$ & $145(19 \%)$ & $447(13 \%)$ & $193(43 \%)$ & $1320(39 \%)$ & $410(31 \%)$ & $1594(41 \%)$ & $491(31 \%)$ \\
\hline Moderate & $5191(46 \%)$ & $2285(44 \%)$ & $3182(52 \%)$ & $1067(34 \%)$ & $1026(50 \%)$ & $209(20 \%)$ & $2064(60 \%)$ & $1080(52 \%)$ & $1515(45 \%)$ & $563(37 \%)$ & $1681(43 \%)$ & $794(47 \%)$ \\
\hline Bad/very bad & $2313(20 \%)$ & $1225(53 \%)$ & $1231(20 \%)$ & $650(53 \%)$ & $263(13 \%)$ & $78(30 \%)$ & $935(27 \%)$ & $724(77 \%)$ & $527(16 \%)$ & $267(51 \%)$ & $625(16 \%)$ & $400(64 \%)$ \\
\hline \multicolumn{13}{|l|}{ Functional Factors } \\
\hline \multicolumn{13}{|l|}{ Mobility } \\
\hline Mobility & $10745(95 \%)$ & $4706(44 \%)$ & $5357(87 \%)$ & $1895(35 \%)$ & $1513(74 \%)$ & $255(17 \%)$ & d & & $\mathrm{d}$ & & $2513(64 \%)$ & $947(38 \%)$ \\
\hline Dismobility & $610(5 \%)$ & $379(62 \%)$ & $814(13 \%)$ & $430(53 \%)$ & $539(26 \%)$ & $177(33 \%)$ & & & & & $1387(36 \%)$ & $738(53 \%)$ \\
\hline \multicolumn{13}{|l|}{ Distance vision impairment } \\
\hline Mild or none & $10054(89 \%)$ & $4422(44 \%)$ & $4888(83 \%)$ & $1783(37 \%)$ & $\mathrm{d}$ & & d & & $2961(88 \%)$ & $1049(35 \%)$ & $3427(88 \%)$ & $1426(42 \%)$ \\
\hline Moderate or greater & $1218(11 \%)$ & $627(52 \%)$ & $997(17 \%)$ & $410(41 \%)$ & & & & & $401(12 \%)$ & $191(48 \%)$ & $473(12 \%)$ & $259(55 \%)$ \\
\hline \multicolumn{13}{|l|}{ Near vision impairment } \\
\hline Mild or none & $7299(64 \%)$ & $3119(43 \%)$ & $3402(57 \%)$ & $1265(37 \%)$ & d & & $\mathrm{d}$ & & $2196(65 \%)$ & $797(36 \%)$ & $2837(73 \%)$ & $1147(40 \%)$ \\
\hline \multirow[t]{2}{*}{ Moderate or greater } & $4056(36 \%)$ & $1966(49 \%)$ & $2521(43 \%)$ & $942(37 \%)$ & & & & & $1166(35 \%)$ & $443(38 \%)$ & $1063(27 \%)$ & $538(51 \%)$ \\
\hline & Mean (SD) & Mean (SD) & Mean (SD) & Mean (SD) & Mean (SD) & Mean (SD) & Mean (SD) & Mean (SD) & Mean (SD) & Mean (SD) & Mean (SD) & Mean (SD) \\
\hline \multicolumn{13}{|l|}{ Verbal learning and memory } \\
\hline Words recalled immediately $\mathrm{e}$ & $5.6(1.6)$ & $5.7(1.7)$ & $5.4(1.4)$ & $5.2(1.4)$ & $5.2(1.4)$ & $5.0(1.5)$ & $6.0(1.6)$ & $5.8(1.6)$ & 5.9 (1.6) & $5.6(1.6)$ & $5.8(1.4)$ & $5.6(1.4)$ \\
\hline Words lost with delay ${ }^{\mathrm{e}}$ & $1.6(1.6)$ & $1.7(1.6)$ & $1.8(1.5)$ & $1.8(1.5)$ & $2.3(2.0)$ & $2.0(1.9)$ & $1.9(1.5)$ & $1.9(1.5)$ & $\mathrm{d}$ & & $1.8(1.6)$ & $1.9(1.6)$ \\
\hline IADL $^{\mathrm{e}}$ & $0.21(0.47)$ & $0.27(0.56)$ & $0.96(0.87)$ & $1.13(0.96)$ & $0.63(0.88)$ & $1.05(1.18)$ & $0.64(0.86)$ & $0.85(0.96)$ & $0.54(0.84)$ & $0.72(0.94)$ & $0.71(0.78)$ & $0.88(0.88)$ \\
\hline $\mathrm{ADL}^{\mathrm{e}}$ & $0.23(0.40)$ & $0.28(0.46)$ & $0.89(0.69)$ & $1.02(0.76)$ & $0.75(0.75)$ & $1.09(0.95)$ & $0.66(0.72)$ & $0.84(0.79)$ & $0.62(0.72)$ & $0.77(0.77)$ & $0.73(0.66)$ & $0.86(0.72)$ \\
\hline Disability $^{\mathrm{e}}$ & $7.3(10.6)$ & $8.6(12.3)$ & $24.0(17.8)$ & $28.2(20.0)$ & $16.3(17.0)$ & $23.8(21.6)$ & $18.3(17.0)$ & $22.7(18.6)$ & $16.5(17.9)$ & $20.6(19.2)$ & $19.4(17.2)$ & $22.8(18.4)$ \\
\hline Quality of Life ${ }^{\mathrm{e}}$ & $65.1(14.2)$ & $64.7(14.9)$ & $61.1(14.8)$ & 57.7 (15.5) & $65.7(12.8)$ & $62.8(13.9)$ & $60.1(15.2)$ & $57.4(15.6)$ & $60.0(15.3)$ & $56.0(15.7)$ & 56.7 (15.5) & $53.4(16.0)$ \\
\hline \multicolumn{13}{|l|}{ Interpersonal Factors } \\
\hline \multicolumn{13}{|l|}{ Emotional loneliness } \\
\hline No & $10645(94 \%)$ & $4751(45 \%)$ & $5167(84 \%)$ & $1926(37 \%)$ & $1689(82 \%)$ & $333(20 \%)$ & $2983(87 \%)$ & $1656(56 \%)$ & $3037(90 \%)$ & $1094(36 \%)$ & $3512(90 \%)$ & $1420(40 \%)$ \\
\hline Yes & $651(6 \%)$ & $305(47 \%)$ & $998(16 \%)$ & $399(40 \%)$ & $363(18 \%)$ & $99(27 \%)$ & $463(13 \%)$ & $341(74 \%)$ & $325(10 \%)$ & $146(45 \%)$ & $388(10 \%)$ & $265(68 \%)$ \\
\hline
\end{tabular}


Table 3. Cont

\begin{tabular}{|c|c|c|c|c|c|c|c|c|c|c|c|c|}
\hline \multirow{3}{*}{ Factors } & \multicolumn{2}{|c|}{ China } & \multicolumn{2}{|c|}{ India } & \multicolumn{2}{|c|}{ Mexico } & \multicolumn{2}{|c|}{ Russian Federation } & \multicolumn{2}{|c|}{ South Africa } & \multicolumn{2}{|c|}{ Ghana } \\
\hline & Sample & Sedentary & Sample & Sedentary & Sample & Sedentary & Sample & Sedentary & Sample & Sedentary & Sample & Sedentary \\
\hline & $\mathrm{n}(\%)^{\mathrm{a}}$ & $n(\%)^{b}$ & $n(\%)^{a}$ & $n(\%)^{b}$ & $n(\%)^{a}$ & $n(\%)^{b}$ & $n(\%)^{a}$ & $n(\%)^{b}$ & $n(\%)^{a}$ & $n(\%)^{b}$ & $n(\%)^{a}$ & $n(\%)^{b}$ \\
\hline \multicolumn{13}{|l|}{ Interpersonal Factors (Cont.) } \\
\hline \multicolumn{13}{|l|}{ Friends visiting home } \\
\hline Never & $3024(27 \%)$ & $1372(45 \%)$ & $1109(18 \%)$ & $470(42 \%)$ & $962(47 \%)$ & $194(20 \%)$ & $455(13 \%)$ & $312(69 \%)$ & $424(13 \%)$ & $162(38 \%)$ & $451(12 \%)$ & $206(46 \%)$ \\
\hline Once or twice per year & $4930(43 \%)$ & $2212(45 \%)$ & $1414(23 \%)$ & $549(39 \%)$ & $418(20 \%)$ & $81(19 \%)$ & $1404(41 \%)$ & $819(58 \%)$ & $413(12 \%)$ & $180(44 \%)$ & $382(10 \%)$ & $159(42 \%)$ \\
\hline Once or twice per month & $2549(22 \%)$ & $1094(43 \%)$ & $1828(30 \%)$ & $632(35 \%)$ & $261(13 \%)$ & $58(22 \%)$ & $1204(35 \%)$ & $660(55 \%)$ & $1003(30 \%)$ & $315(31 \%)$ & $790(20 \%)$ & $370(47 \%)$ \\
\hline Once per week or more & $852(8 \%)$ & $407(48 \%)$ & $1820(30 \%)$ & $674(37 \%)$ & $411(20 \%)$ & $99(24 \%)$ & $374(11 \%)$ & $201(54 \%)$ & $1522(45 \%)$ & $583(38 \%)$ & $2277(58 \%)$ & $950(42 \%)$ \\
\hline \multicolumn{13}{|l|}{ Visiting people ${ }^{f}$} \\
\hline Never & $4003(35 \%)$ & $1933(48 \%)$ & $978(16 \%)$ & $481(49 \%)$ & $1061(52 \%)$ & $227(21 \%)$ & $1035(30 \%)$ & $677(65 \%)$ & $575(17 \%)$ & $234(41 \%)$ & $662(17 \%)$ & $326(49 \%)$ \\
\hline Once or twice per year & $4951(44 \%)$ & $2095(42 \%)$ & $1728(28 \%)$ & $683(40 \%)$ & $469(23 \%)$ & $85(18 \%)$ & $1508(44 \%)$ & $847(56 \%)$ & $608(18 \%)$ & $219(36 \%)$ & $534(14 \%)$ & $283(53 \%)$ \\
\hline Once or twice per month & $1635(14 \%)$ & $704(43 \%)$ & $1706(28 \%)$ & $584(34 \%)$ & $266(13 \%)$ & $56(21 \%)$ & $736(21 \%)$ & $393(53 \%)$ & $1109(33 \%)$ & $393(35 \%)$ & $963(25 \%)$ & $449(47 \%)$ \\
\hline Once per week or more & $766(7 \%)$ & $353(46 \%)$ & $1759(29 \%)$ & $577(33 \%)$ & $256(13 \%)$ & $64(25 \%)$ & $153(5 \%)$ & $72(47 \%)$ & $1061(32 \%)$ & $390(37 \%)$ & $1741(45 \%)$ & $627(36 \%)$ \\
\hline \multicolumn{13}{|l|}{ Socializing with coworkers } \\
\hline Never & $3182(28 \%)$ & $1526(48 \%)$ & $2540(41 \%)$ & $957(38 \%)$ & $1504(73 \%)$ & $333(22 \%)$ & $1658(48 \%)$ & $1093(66 \%)$ & $\mathrm{d}$ & & $1604(41 \%)$ & $844(53 \%)$ \\
\hline Once or twice per year & $2860(25 \%)$ & $1271(44 \%)$ & $1416(23 \%)$ & $567(40 \%)$ & $280(14 \%)$ & $41(15 \%)$ & $806(23 \%)$ & $425(53 \%)$ & & & $389(10 \%)$ & $167(43 \%)$ \\
\hline Once or twice per month & $2530(22 \%)$ & $1080(43 \%)$ & $1102(18 \%)$ & $394(36 \%)$ & $142(7 \%)$ & $29(20 \%)$ & $562(16 \%)$ & $276(49 \%)$ & & & $602(15 \%)$ & $184(31 \%)$ \\
\hline Once per week or more & $2778(25 \%)$ & $1206(43 \%)$ & $1113(18 \%)$ & $407(37 \%)$ & $126(6 \%)$ & $29(23 \%)$ & $420(12 \%)$ & $203(48 \%)$ & & & $1305(34 \%)$ & $490(38 \%)$ \\
\hline \multicolumn{13}{|c|}{ Social activities outside of home } \\
\hline Never & $2130(19 \%)$ & $1018(48 \%)$ & $1319(21 \%)$ & $528(40 \%)$ & $960(47 \%)$ & $192(20 \%)$ & $1244(36 \%)$ & $744(60 \%)$ & $789(24 \%)$ & $274(35 \%)$ & $505(13 \%)$ & $225(45 \%)$ \\
\hline Once or twice per year & $6767(60 \%)$ & $2881(43 \%)$ & $3424(56 \%)$ & $1327(39 \%)$ & $630(31 \%)$ & $155(25 \%)$ & $1003(29 \%)$ & $597(60 \%)$ & $753(22 \%)$ & $265(35 \%)$ & $668(17 \%)$ & $283(42 \%)$ \\
\hline Once or twice per month & $2002(18 \%)$ & $928(46 \%)$ & $1133(18 \%)$ & $374(33 \%)$ & $292(14 \%)$ & $56(19 \%)$ & $710(21 \%)$ & $385(54 \%)$ & $1024(31 \%)$ & $375(37 \%)$ & $994(26 \%)$ & $550(55 \%)$ \\
\hline Once per week or more & $456(4 \%)$ & $258(57 \%)$ & $294(5 \%)$ & $95(32 \%)$ & $170(8 \%)$ & $29(17 \%)$ & $483(14 \%)$ & $266(55 \%)$ & $796(24 \%)$ & $326(41 \%)$ & $1733(44 \%)$ & $627(36 \%)$ \\
\hline \multicolumn{13}{|l|}{ Marital status } \\
\hline Coupled & $9488(84 \%)$ & $4123(44 \%)$ & $4631(75 \%)$ & $1651(36 \%)$ & $1252(61 \%)$ & $244(20 \%)$ & $1954(57 \%)$ & $1035(53 \%)$ & $1784(53 \%)$ & $599(34 \%)$ & $2224(57 \%)$ & $879(40 \%)$ \\
\hline Uncoupled & $1867(16 \%)$ & $962(52 \%)$ & $1540(25 \%)$ & $674(44 \%)$ & $800(39 \%)$ & $188(24 \%)$ & $1492(43 \%)$ & $962(65 \%)$ & $1578(47 \%)$ & $641(41 \%)$ & $1676(43 \%)$ & $806(48 \%)$ \\
\hline \multicolumn{13}{|l|}{ Environmental Factors } \\
\hline \multicolumn{13}{|l|}{ Location } \\
\hline Urban & $5347(47 \%)$ & $2859(54 \%)$ & $1535(25 \%)$ & $597(39 \%)$ & $1478(72 \%)$ & $348(24 \%)$ & $2608(76 \%)$ & $1537(59 \%)$ & $2247(67 \%)$ & $820(37 \%)$ & $1568(40 \%)$ & $735(47 \%)$ \\
\hline Rural & $6008(53 \%)$ & $2226(37 \%)$ & $4636(75 \%)$ & $1728(37 \%)$ & $574(28 \%)$ & $84(15 \%)$ & $838(24 \%)$ & $460(55 \%)$ & $1114(33 \%)$ & $419(38 \%)$ & $2332(60 \%)$ & $950(41 \%)$ \\
\hline \multicolumn{13}{|l|}{ Personal motorized transport } \\
\hline No & $5165(46 \%)$ & $2189(42 \%)$ & $4564(74 \%)$ & $1773(39 \%)$ & $1308(64 \%)$ & $278(21 \%)$ & $1966(57 \%)$ & $1233(63 \%)$ & $2475(74 \%)$ & $980(40 \%)$ & $3685(95 \%)$ & $1596(43 \%)$ \\
\hline Yes & $6190(55 \%)$ & $2896(47 \%)$ & $1607(26 \%)$ & $552(34 \%)$ & $743(36 \%)$ & $153(21 \%)$ & $1480(43 \%)$ & $764(52 \%)$ & $887(26 \%)$ & $260(29 \%)$ & $215(6 \%)$ & $89(41 \%)$ \\
\hline \multicolumn{13}{|l|}{ Computer in household } \\
\hline & $8827(78 \%)$ & $3736(42 \%)$ & $5873(95 \%)$ & $2213(38 \%)$ & $1737(85 \%)$ & $354(20 \%)$ & $2782(81 \%)$ & $1649(59 \%)$ & $2894(86 \%)$ & $1100(38 \%)$ & $3714(96 \%)$ & $1603(43 \%)$ \\
\hline Yes & $2528(22 \%)$ & $1349(53 \%)$ & $298(5 \%)$ & $112(38 \%)$ & $314(15 \%)$ & $77(25 \%)$ & $664(19 \%)$ & $348(52 \%)$ & $468(14 \%)$ & $140(30 \%)$ & $177(5 \%)$ & $76(43 \%)$ \\
\hline \multicolumn{13}{|c|}{ Safety out on the street after dark } \\
\hline Completely/very safe & $6806(60 \%)$ & $2992(44 \%)$ & $3763(61 \%)$ & $1583(42 \%)$ & $724(35 \%)$ & $154(21 \%)$ & $537(16 \%)$ & $291(54 \%)$ & $508(15 \%)$ & $233(46 \%)$ & $2851(73 \%)$ & $1134(40 \%)$ \\
\hline Moderately safe & $2947(26 \%)$ & $1343(46 \%)$ & $1467(24 \%)$ & $463(32 \%)$ & $501(24 \%)$ & $101(20 \%)$ & $1005(30 \%)$ & $555(55 \%)$ & $648(19 \%)$ & $268(41 \%)$ & $719(18 \%)$ & $384(53 \%)$ \\
\hline Slightly safe/not at all & $1591(14 \%)$ & $746(47 \%)$ & $941(15 \%)$ & $279(30 \%)$ & $827(40 \%)$ & $177(21 \%)$ & $1865(55 \%)$ & $1125(60 \%)$ & $2206(66 \%)$ & $739(34 \%)$ & $330(9 \%)$ & $167(51 \%)$ \\
\hline
\end{tabular}


Table 3. Cont

\begin{tabular}{|c|c|c|c|c|c|c|c|c|c|c|c|c|}
\hline \multirow{3}{*}{ Factors } & \multicolumn{2}{|c|}{ China } & \multicolumn{2}{|c|}{ India } & \multicolumn{2}{|c|}{ Mexico } & \multicolumn{2}{|c|}{ Russian Federation } & \multicolumn{2}{|c|}{ South Africa } & \multicolumn{2}{|c|}{ Ghana } \\
\hline & Sample & Sedentary & Sample & Sedentary & Sample & Sedentary & Sample & Sedentary & Sample & Sedentary & Sample & Sedentary \\
\hline & $n(\%)^{a}$ & $n(\%)^{b}$ & $n(\%)^{a}$ & $n(\%)^{b}$ & $n(\%)^{a}$ & $n(\%)^{b}$ & $n(\%)^{a}$ & $n(\%)^{b}$ & $n(\%)^{a}$ & $n(\%)^{b}$ & $n(\%)^{a}$ & $n(\%)^{b}$ \\
\hline \multicolumn{13}{|c|}{ Environmental Factors (Cont.) } \\
\hline Safety when home alone & & & & & & & & & & & & \\
\hline Completely/very safe & $9527(84 \%)$ & $4250(45 \%)$ & $4147(67 \%)$ & $1687(41 \%)$ & $1139(56 \%)$ & $255(22 \%)$ & $1494(43 \%)$ & $825(55 \%)$ & $801(24 \%)$ & $345(43 \%)$ & $3306(85 \%)$ & $1413(43 \%)$ \\
\hline Moderately safe & $1482(13 \%)$ & $681(46 \%)$ & $1457(24 \%)$ & $455(31 \%)$ & $424(21 \%)$ & $87(21 \%)$ & $1132(33 \%)$ & $645(57 \%)$ & $917(27 \%)$ & $338(37 \%)$ & $425(11 \%)$ & $192(45 \%)$ \\
\hline Slightly safe/not at all & $339(3 \%)$ & $151(45 \%)$ & $567(9 \%)$ & $183(32 \%)$ & $489(24 \%)$ & $90(18 \%)$ & $820(24 \%)$ & $527(64 \%)$ & $1642(49 \%)$ & $556(34 \%)$ & $169(4 \%)$ & $80(47 \%)$ \\
\hline
\end{tabular}

${ }^{a}$ Distribution of the factors in each country's sample. ${ }^{b}$ Percentages of people sedentary of $\geq 4 \mathrm{~h} /$ day for each factor level. ${ }^{c}$ Education levels for some countries collapsed (e.g., secondary/high and college or more for India) due to low frequencies. ${ }^{\mathrm{d}}$ Factor omitted from the analyses due to large amounts of missing or implausible data. ${ }^{\mathrm{e}}$ Range: $0-10$ for words recalled immediately (higher scores $=$ higher function) and words lost with delay (higher scores $=$ lower function), $0-5$ for IADL and ADL (higher scores $=$ lower function), and $0-100$ for disability (higher scores $=$ lower function) and quality of life (higher scores $=$ higher function). ${ }^{\mathrm{f}}$ Visiting people in different neighborhoods or them coming to visit.

Table 4. Multivariate associations of $\geq 4 \mathrm{~h} /$ day sedentary behavior with demographic, health and health risk, functional, interpersonal, and environmental factors.

\begin{tabular}{|c|c|c|c|c|c|c|c|c|c|c|c|c|}
\hline \multirow{2}{*}{ Factors } & \multicolumn{2}{|c|}{ China } & \multicolumn{2}{|c|}{ India } & \multicolumn{2}{|c|}{ Mexico } & \multicolumn{2}{|c|}{ Russian Federation } & \multicolumn{2}{|c|}{ South Africa } & \multicolumn{2}{|c|}{ Ghana } \\
\hline & OR $(95 \% \mathrm{CI})^{\mathrm{a}}$ & $p^{a}$ & $\mathrm{OR}(95 \% \mathrm{CI})^{\mathrm{a}}$ & $p^{a}$ & OR $(95 \% \mathrm{CI})^{\mathrm{a}}$ & $p^{a}$ & $\mathrm{OR}(95 \% \mathrm{CI})^{\mathrm{a}}$ & $p^{\mathrm{a}}$ & OR $(95 \% \mathrm{CI})^{\mathrm{a}}$ & $p^{a}$ & OR $(95 \% \mathrm{CI})^{\mathrm{a}}$ & $p^{a}$ \\
\hline \multicolumn{13}{|l|}{ Demographic Factors } \\
\hline \multicolumn{13}{|l|}{ Age (Ref: 50-59) } \\
\hline $60-69$ & $1.22(1.10,1.35)$ & $<0.0001$ & $1.05(0.92,1.20)$ & 0.0070 & $1.20(0.85,1.71)$ & 0.0139 & $1.24(1.02,1.52)$ & $<0.0001$ & $1.12(0.93,1.36)$ & 0.0802 & $1.00(0.83,1.19)$ & 0.0109 \\
\hline $70-79$ & $1.56(1.37,1.77)$ & & $1.29(1.09,1.54)$ & & $1.43(0.98,2.10)$ & & $1.25(0.99,1.59)$ & & $1.35(1.07,1.71)$ & & $1.25(1.02,1.54)$ & \\
\hline $80+$ & $2.16(1.73,2.71)$ & & $1.44(1.08,1.92)$ & & $2.07(1.30,3.28)$ & & $2.58(1.80,3.75)$ & & $1.26(0.90,1.76)$ & & $1.52(1.13,2.06)$ & \\
\hline \multicolumn{13}{|l|}{ Sex (Ref: Male) } \\
\hline Female & $1.03(0.94,1.13)$ & 0.5679 & $0.72(0.62,0.83)$ & $<0.0001$ & $0.71(0.54,0.93)$ & 0.0131 & $0.94(0.79,1.11)$ & 0.4550 & $0.92(0.77,1.08)$ & 0.2961 & $1.12(0.94,1.35)$ & 0.2135 \\
\hline \multicolumn{13}{|c|}{ Education completed (Ref: Lowest level, country-specific) ${ }^{\mathrm{b}}$} \\
\hline Less than primary school & $1.02(0.90,1.16)$ & 0.0042 & c & & $1.15(0.85,1.58)$ & 0.0415 & & & d & & & \\
\hline Primary school & $1.06(0.93,1.21)$ & & & & $0.98(0.67,1.43)$ & & & & & & $1.06(0.84,1.35)$ & 0.7595 \\
\hline Secondary/high school & $1.18(1.03,1.35)$ & & & & $1.82(1.16,2.85)$ & & $0.79(0.59,1.03)$ & 0.0010 & & & $0.96(0.79,1.17)$ & \\
\hline College or more & $1.50(1.20,1.90)$ & & & & $1.41(0.88,2.24)$ & & $1.09(0.79,1.50)$ & & & & & \\
\hline \multicolumn{13}{|l|}{ Employment (Ref: Working) } \\
\hline Not working & $1.51(1.35,1.69)$ & $<0.0001$ & $1.97(1.70,2.28)$ & $<0.0001$ & $0.99(0.73,1.35)$ & 0.0759 & $1.12(0.87,1.44)$ & 0.6011 & $1.66(1.35,2.04)$ & $<0.0001$ & $1.67(1.36,2.04)$ & $<0.000$ \\
\hline Retired/too old to work & $1.30(1.16,1.46)$ & & $2.20(1.83,2.64)$ & & $1.52(0.98,2.34)$ & & $1.09(0.89,1.35)$ & & $1.72(1.37,2.17)$ & & $2.05(1.60,2.65)$ & \\
\hline \multicolumn{13}{|c|}{ Household wealth (Ref: 1st (high) quintile) } \\
\hline 2nd & $0.95(0.83,1.09)$ & $<0.0001$ & $0.66(0.55,0.79)$ & 0.0001 & c & & c & & $0.77(0.59,1.02)$ & 0.0122 & $0.98(0.78,1.22)$ & 0.2742 \\
\hline $3 \mathrm{rd}$ & $0.70(0.59,0.82)$ & & $0.71(0.58,0.88)$ & & & & & & $0.82(0.60,1.11)$ & & $0.82(0.64,1.04)$ & \\
\hline 4th & $0.60(0.50,0.71)$ & & $0.76(0.61,0.93)$ & & & & & & $1.11(0.81,1.52)$ & & $0.92(0.71,1.18)$ & \\
\hline 5th (low) quintile & $0.47(0.39,0.57)$ & & $0.66(0.53,0.83)$ & & & & & & $0.83(0.60,1.15)$ & & $1.03(0.79,1.34)$ & \\
\hline \multicolumn{13}{|c|}{ Health and Health-Risk Factors } \\
\hline \multicolumn{13}{|c|}{ Body mass index (Ref: Normal weight) } \\
\hline Underweight & $1.12(0.93,1.36)$ & $<0.0001$ & $1.03(0.90,1.16)$ & 0.8483 & d & & $\mathrm{d}$ & & $\mathrm{d}$ & & $1.04(0.85,1.28)$ & 0.0445 \\
\hline Overweight & $1.24(1.13,1.35)$ & & $0.94(0.78,1.13)$ & & & & & & & & $1.21(0.99,1.46)$ & \\
\hline Obese & $1.36(1.14,1.63)$ & & $0.97(0.69,1.35)$ & & & & & & & & $1.40(1.08,1.82)$ & \\
\hline
\end{tabular}


Table 4. Cont

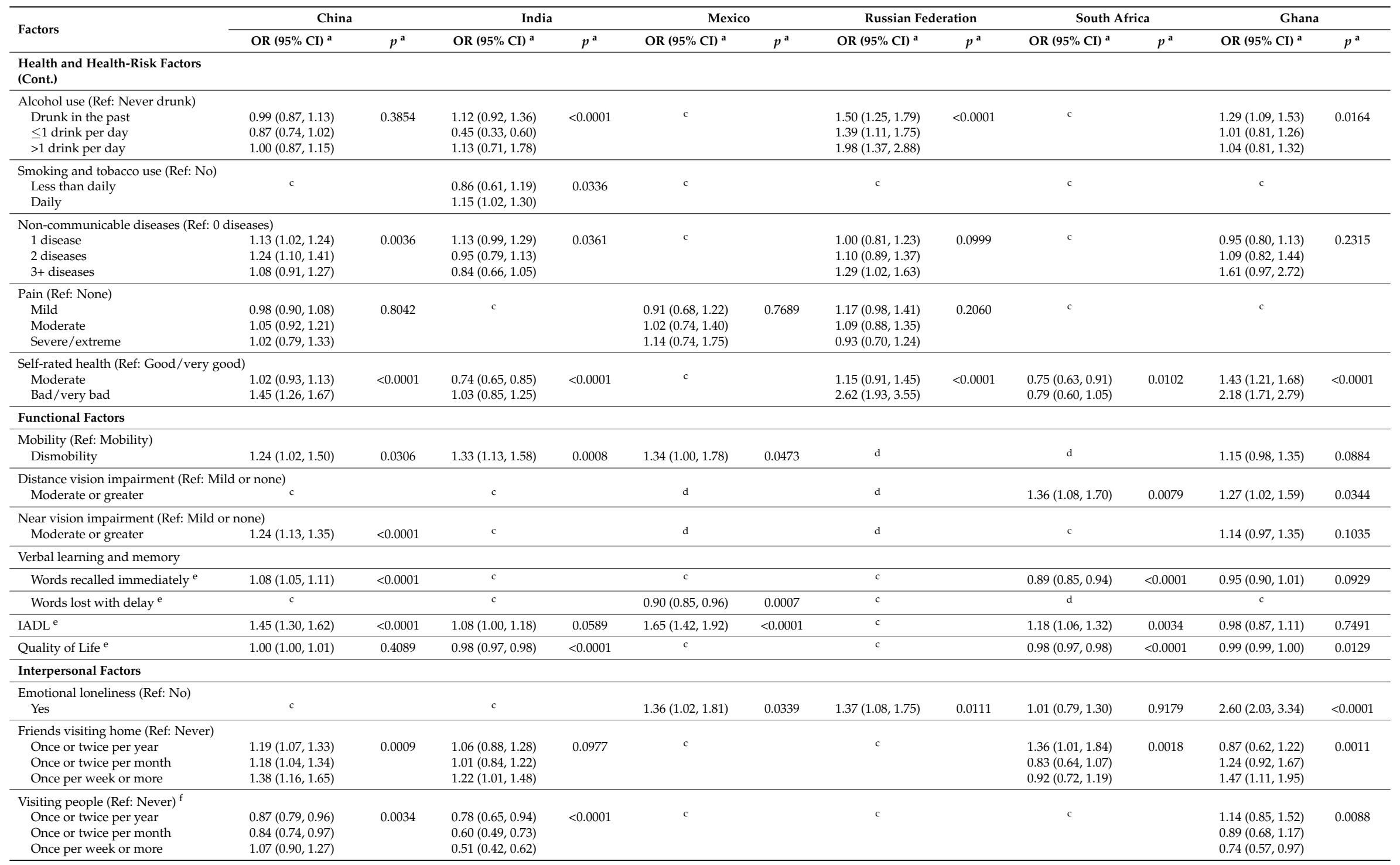


Table 4. Cont.

\begin{tabular}{|c|c|c|c|c|c|c|c|c|c|c|c|c|}
\hline \multirow{2}{*}{ Factors } & \multicolumn{2}{|c|}{ China } & \multicolumn{2}{|c|}{ India } & \multicolumn{2}{|c|}{ Mexico } & \multicolumn{2}{|c|}{ Russian Federation } & \multicolumn{2}{|c|}{ South Africa } & \multicolumn{2}{|c|}{ Ghana } \\
\hline & OR $(95 \% \mathrm{CI})^{\mathrm{a}}$ & $p^{\mathrm{a}}$ & OR $(95 \% \mathrm{CI})^{\mathrm{a}}$ & $p^{\mathrm{a}}$ & OR $(95 \% \mathrm{CI})^{\mathrm{a}}$ & $p^{\mathrm{a}}$ & OR $(95 \% \mathrm{CI})^{\mathrm{a}}$ & $p^{\mathrm{a}}$ & OR $(95 \% \mathrm{CI})^{\mathrm{a}}$ & $p^{\mathrm{a}}$ & OR $(95 \% \mathrm{CI})^{\mathrm{a}}$ & $p^{\mathrm{a}}$ \\
\hline \multicolumn{13}{|l|}{ Interpersonal Factors (Cont.) } \\
\hline \multicolumn{13}{|c|}{ Socializing with coworkers (Ref: Never) } \\
\hline Once or twice per year & c & & $1.34(1.15,1.56)$ & 0.0003 & $0.63(0.43,0.91)$ & 0.0343 & $0.80(0.66,0.97)$ & 0.0437 & $\mathrm{~d}$ & & $0.91(0.71,1.18)$ & $<0.0001$ \\
\hline Once or twice per month & & & $1.27(1.07,1.50)$ & & $1.08(0.68,1.68)$ & & $0.78(0.63,0.98)$ & & & & $0.48(0.38,0.60)$ & \\
\hline Once per week or more & & & $1.32(1.12,1.57)$ & & $1.37(0.85,2.17)$ & & $0.78(0.61,1.00)$ & & & & $0.78(0.65,0.94)$ & \\
\hline \multicolumn{3}{|c|}{ Social activities outside of home (Ref: Never) } & & & & & & & & & & \\
\hline Once or twice per year & $0.96(0.87,1.08)$ & 0.0005 & c & & c & & c & & $1.20(0.95,1.51)$ & $<0.0001$ & $1.29(0.98,1.70)$ & $<0.0001$ \\
\hline Once or twice per month & $1.04(0.91,1.19)$ & & & & & & & & $1.56(1.26,1.94)$ & & $2.43(1.86,3.19)$ & \\
\hline Once per week or more & $1.48(1.19,1.84)$ & & & & & & & & $1.61(1.27,2.04)$ & & $1.37(1.06,1.77)$ & \\
\hline \multicolumn{13}{|l|}{ Marital status (Ref: Coupled) } \\
\hline Uncoupled & $1.18(1.05,1.32)$ & 0.0043 & $1.13(0.99,1.30)$ & 0.0759 & $1.04(0.80,1.34)$ & 0.7628 & $1.17(0.99,1.38)$ & 0.0708 & $1.09(0.92,1.28)$ & 0.3396 & $0.87(0.72,1.04)$ & 0.1171 \\
\hline \multicolumn{13}{|l|}{ Environmental Factors } \\
\hline \multicolumn{12}{|l|}{ Location (Ref: Urban) } & \\
\hline Rural & $0.73(0.65,0.81)$ & $<0.0001$ & c & & $0.56(0.41,0.74)$ & $<0.0001$ & c & & c & & $0.89(0.75,1.05)$ & 0.1615 \\
\hline \multicolumn{13}{|c|}{ Personal motorized transport (Ref: No) } \\
\hline Yes & $0.88(0.79,0.97)$ & 0.0112 & $0.80(0.67,0.94)$ & 0.0071 & c & & $0.85(0.73,0.99)$ & 0.0394 & $0.80(0.63,1.01)$ & 0.059 & c & \\
\hline \multicolumn{13}{|c|}{ Computer in household (Ref: No) } \\
\hline Yes & $1.00(0.88,1.15)$ & 0.9579 & c & & c & & $1.07(0.88,1.30)$ & 0.4848 & $1.00(0.77,1.30)$ & 0.986 & c & \\
\hline \multicolumn{13}{|c|}{ Safety out on the street after dark (Ref: Completely/very safe) } \\
\hline $\begin{array}{l}\text { Moderately safe } \\
\text { Slightly safe/not at all }\end{array}$ & & & $\begin{array}{l}0.58(0.50,0.66) \\
0.50(0.42,0.59)\end{array}$ & $<0.0001$ & c & & c & & $\begin{array}{l}0.76(0.59,0.97) \\
0.55(0.44,0.68)\end{array}$ & $<0.0001$ & $\begin{array}{l}1.39(1.15,1.68) \\
1.31(1.01,1.69)\end{array}$ & 0.0010 \\
\hline \multirow{2}{*}{\multicolumn{3}{|c|}{$\begin{array}{l}\text { Safety when home alone (Ref: Completely/very safe) } \\
\text { Moderately safe }\end{array}$}} & & & & & & & & & & \\
\hline & & & c & & c & & $\begin{array}{l}1.03(0.87,1.22) \\
1.19(0.98,1.44)\end{array}$ & 0.1886 & c & & c & \\
\hline
\end{tabular}

${ }^{\mathrm{a}}$ Odds ratios (95\% confidence intervals) and $\mathrm{p}$ values for those factors that were selected in the fully adjusted final model for each country. ${ }^{\mathrm{b}}$ Education levels for some countries collapsed (e.g., secondary/high and college or more for India) due to low numbers. ${ }^{\mathrm{c}}$ Factor was not selected for the final model. ${ }^{\mathrm{d}}$ Factor omitted from the analyses due to large amounts of missing or implausible data. ${ }^{\mathrm{e}}$ Higher scores $=$ higher function for words recalled immediately and quality of life; higher scores $=$ lower function for words lost with delay and IADL. ${ }^{\mathrm{f}}$ Visiting people in different neighborhoods or them coming to visit. 


\subsubsection{Environmental Factors}

Adults in India and South Africa who perceived themselves to be completely or very safe out on the street after dark had higher odds of $\geq 4 \mathrm{~h}$ /day sedentary behavior than those who perceived themselves to be moderately safe or slightly safe/not at all safe. The reverse was observed for Ghana. Those with access to personal motorized transport had lower odds of $\geq 4 \mathrm{~h}$ /day sedentary behavior in China, India, and Russian Federation, with a nonsignificant association in the same direction for South Africa. People in China and Mexico living in rural areas had lower odds of $\geq 4 \mathrm{~h}$ /day sedentary behavior. No significant associations were present between $\geq 4 \mathrm{~h}$ /day sedentary behavior and either computers in household or safety when home alone.

\section{Discussion}

Across the six LMIC, we found fairly consistent and reasonably strong associations (fully adjusted models) between both physical activity and sedentary behavior and demographic factors (age and employment, in particular), self-reported health, IADL, factors relating to socializing, and location of household. Age was the only variable that was related to both physical activity and sedentary behavior in all six countries. Like in previous research [4,12], age had an inverse relationship with physical activity and a positive association with sedentary behavior. Some researchers have speculated that this decline in activity with age may have a biological basis (e.g., reduced dopamine release or loss of dopamine receptors with age, affecting areas of the brain relating to motivation for locomotion) in conjunction with psychosocial factors and the physical environment [39-41]. Across countries, older adults who were working were clearly more active than those who were not working or were retired/too old to work. This finding suggests that the work-related physical activity (e.g., active transport to and from work, and occupation-related physical activity) is not replaced with other activity (e.g., leisure time physical activity) for older adults who have retired or are otherwise not working. The associations between work and sedentary behavior are consistent with the findings of studies from (mainly) high-income countries [12].

Of the health and health-risk factors, self-rated health was the factor most consistently linked with physical activity and sedentary behavior. Poorer rated health was associated with lower odds of meeting physical activity guidelines and higher odds of sedentary behavior. The prominence of this factor lends itself to at least two possible interpretations. First, self-reported health may have captured aspects of health not identified through the other factors (e.g., undiagnosed non-communicable diseases and health conditions not included in the SAGE dataset). Second, individuals' perceptions of health may matter more than the views of researchers (who decide what health conditions are included in surveys) and health practitioners as to what constitutes good health. Using self-rated health measures in surveys enables participants to prioritize and assess different aspects of their health, which is perhaps a more sensitive way of measuring general health [42].

The findings pertaining to functional factors serve to re-emphasize the positive relationship between physical function and physical activity. Across all countries, with the exception of Russian Federation, having fewer difficulties with IADL was associated with increased odds of meeting physical activity guidelines, and in three countries (China, Mexico, and South Africa), greater difficulties with IADL was correlated with sedentary behavior. The relationship between activity and function may be bidirectional (i.e., increased physical activity may improve physical function, and higher levels of physical function may increase a person's willingness to be physically active). Evidence from studies with community-dwelling older adults is that increased levels of physical activity are associated with fewer difficulties with basic activities of daily living [43]. Equally, greater physical function promotes increased participation in physical activity [44].

Taken collectively, the results for interpersonal factors generally show that more frequent social activity is associated with increased odds of meeting physical activity guidelines and decreased likelihood of spending $\geq 4 \mathrm{~h}$ /day sedentary. In general, these findings would seem to broadly reflect the literature, in which an association between social support and physical activity in older adults has 
been reported [45]. This relationship would appear to be stronger in some circumstances (e.g., when the social support is for physical activity and comes from family members) than others (e.g., when general social support is provided). In our study, the reasons for the relationships were not evident. Plausible explanations include: (a) participating in social activities required active transport to and from the activities; (b) the social activities involved physical activity; and (c) those who were more physically active may have been more willing and able to attend the social activities.

Of the environmental factors, where an older adult lived (urban versus rural) had the most consistent relationship with physical activity and sedentary behavior. Adults in rural areas were generally more likely to be meeting physical activity guidelines and less likely to be spending $\geq 4 \mathrm{~h}$ /day sedentary. These results confirm previous findings linking urbanization with lower physical activity and greater sedentary behavior [5,7].

Much higher levels of physical inactivity (not meeting physical activity guidelines) were found in the SAGE data than in a previous study of 51 mainly LMIC included in the World Health Survey (2001) [3]. In this World Health Survey study and our study, findings can be compared for people aged 60-69 years. For this age group, the levels of inactivity (the inverse of the findings for meeting physical activity guidelines in Table 1) were higher in our study with SAGE data (China, 68\%; India, 62\%; Mexico, 68\%; Russian Federation, 50\%; South Africa, 84\%; Ghana, 38\%) than in the World Health Survey (22\% for men and 29\% for women). The researchers using World Health Survey study data recognized the prevalence of physical inactivity among people in this survey was substantially lower than in comparable studies, and suggested instrumentation and the seasons in which data were collected may have influenced results. The results from SAGE data are consistent with evidence from a systematic review that between $20 \%$ and $60 \%$ of older adults (aged 60 and over) have typically been found to meet physical activity guidelines (i.e., between $40 \%$ and $80 \%$ are physically inactive) [46].

A small number of associations seemed counterintuitive. Notably, in the fully adjusted models of three countries, people with more severe pain had higher odds of meeting physical activity guidelines. The direction of this relationship was opposite to that found in the unadjusted models. Overadjustment or misspecification of the models may explain these unexpected findings.

This study has several limitations. First, although physical activity questionnaires enable the timely and cost-effective gathering of data in large epidemiological studies, the limitations of such measures are well known $[47,48]$. Pertinent to our study is the appropriateness of the GPAQ for research with older adults from several LMIC. There is evidence that older adults sometimes find questions on physical activity and sedentary behavior difficult to understand, often resulting in misreporting (overreporting more commonly than underreporting) of activity levels [49]. Such issues may also affect younger adults. The implications of these biases are that some older adults in our study may have been miscategorized (e.g., classified as meeting physical activity guidelines when they, in actuality, performed insufficient moderate and vigorous physical activity to meet the threshold). Such misclassification bias may have attenuated some of the estimated associations. In addition, although the GPAQ has been evaluated and used in many developed and developing countries [33,50], the potential remains for the differences observed between countries to be an artifact of differences between various translations of the GPAQ and how questions were asked, interpreted, and answered. Second, the SAGE survey involved a long interview that might have affected the accuracy of responses, especially in older adults who are frail. Third, this study had a cross-sectional design, meaning that temporality between the variables cannot be established. Fourth, the response rates for Mexico were much lower than for the other five countries, potentially underrepresenting some subpopulations for this country. The lower response rates for Mexico have been attributed to the short time available for fieldwork (data were collected from 2009 to 2010 and multiple visits to respondents' homes were not made if they were not at home during the initial visit) and a high level of attrition from Wave 0 [14]. Wave 0 in SAGE refers to data collected from the six countries during the 2002/2004 World Health Survey. Even with these limitations, to our knowledge this is the first study to report on the correlates of physical activity and sedentary behavior for representative samples of older adults in several LMIC. 


\section{Conclusions}

The central finding from our work is that physical activity and sedentary behavior were fairly consistently related to several demographic factors (age and employment, in particular), self-reported health, IADL, factors relating to socializing, and location of household. Our findings may be useful in targeting interventions towards those who are most likely to be physically inactive. Thought needs to be given to developing interventions that target adults with specific characteristics (e.g., those who are older) and to do so using methods that are sensitive to the resource constraints of LMIC. Further research is necessary to understand how these factors may interact and which of them may be modifiable determinants. We expect that analysis of data from the next two waves of SAGE (when they become available) will yield valuable information in this respect. In the design of future projects, strong consideration should be given to using objective measures of physical activity and sedentary behavior. The use of such measures would strengthen research findings.

Supplementary Materials: The following tables are available online at http:/ / www.mdpi.com/1660-4601/15/5/ 908/s1, Table S1: Univariate associations of meeting physical activity guidelines with demographic, health and health risk, functional, interpersonal, and environmental factors, Table S2: Univariate associations of $\geq 4 \mathrm{~h} /$ day sedentary behavior with demographic, health and health risk, functional, interpersonal, and environmental factors.

Author Contributions: C.J.G. and L.O. conceived the study, analyzed the data, and wrote the paper.

Acknowledgments: The authors thank all the respondents who contributed to SAGE and the survey teams. No specific financial support was provided for this analysis. SAGE is supported by WHO and the US National Institute on Aging through Interagency Agreements (OGHA 04034785; YA1323-08-CN-0020; Y1-AG-1005-01) and through a research grant (R01-AG034479).

Conflicts of Interest: The founding sponsors had no role in the design of the study; in the collection, analyses, or interpretation of data; in the writing of the manuscript, and in the decision to publish the results.

\section{References}

1. World Health Organization. Global Status Report on Noncommunicable Diseases 2010; WHO: Geneva, Switzerland, 2011.

2. World Health Organization. Global Status Report on Noncommunicable Diseases 2014; WHO: Geneva, Switzerland, 2014.

3. Guthold, R.; Ono, T.; Strong, K.L.; Chatterji, S.; Morabia, A. Worldwide variability in physical inactivity: A 51-country survey. Am. J. Prev. Med. 2008, 34, 486-494. [CrossRef] [PubMed]

4. Bauman, A.E.; Reis, R.S.; Sallis, J.F.; Wells, J.C.; Loos, R.J.F.; Martin, B.W. Correlates of physical activity: Why are some people physically active and others not? Lancet 2012, 380, 258-271. [CrossRef]

5. Moniruzzaman, M.; Ahmed, M.S.A.M.; Zaman, M.M. Physical activity levels and associated sociodemographic factors in Bangladeshi adults: A cross-sectional study. BMC Public Health 2017, 17, 59. [CrossRef] [PubMed]

6. Pengpid, S.; Peltzer, K.; Kassean, H.K.; Tsala Tsala, J.P.; Sychareun, V.; Müller-Riemenschneider, F. Physical inactivity and associated factors among university students in 23 low-, middle- and high-income countries. Int. J. Public Health 2015, 60, 539-549. [CrossRef] [PubMed]

7. Attard, S.M.; Howard, A.-G.; Herring, A.H.; Zhang, B.; Du, S.; Aiello, A.E.; Popkin, B.M.; Gordon-Larsen, P. Differential associations of urbanicity and income with physical activity in adults in urbanizing China: Findings from the population-based China Health and Nutrition Survey 1991-2009. Int. J. Behav. Nutr. Phys. Act. 2015, 12, 152. [CrossRef] [PubMed]

8. Lear, S.A.; Teo, K.; Gasevic, D.; Zhang, X.; Poirier, P.P.; Rangarajan, S.; Seron, P.; Kelishadi, R.; Tamil, A.M.; Kruger, A.; et al. The association between ownership of common household devices and obesity and diabetes in high, middle and low income countries. Can. Med. Assoc. J. 2014, 186, 258-266. [CrossRef] [PubMed]

9. Salvo, D.; Torres, C.; Villa, U.; Rivera, J.A.; Sarmiento, O.L.; Reis, R.S.; Pratt, M. Accelerometer-based physical activity levels among Mexican adults and their relation with sociodemographic characteristics and BMI: A cross-sectional study. Int. J. Behav. Nutr. Phys. Act. 2015, 12, 79. [CrossRef] [PubMed]

10. Hino, A.A.F.; Reis, R.S.; Sarmiento, O.L.; Parra, D.C.; Brownson, R.C. Built environment and physical activity for transportation in adults from Curitiba, Brazil. J. Urban Health 2014, 91, 446-462. [CrossRef] [PubMed] 
11. Du, H.; Bennett, D.; Li, L.; Whitlock, G.; Guo, Y.; Collins, R.; Chen, J.; Bian, Z.; Hong, L.-S.; Feng, S.; et al. Physical activity and sedentary leisure time and their associations with BMI, waist circumference, and percentage body fat in 0.5 million adults: The China Kadoorie Biobank study. Am. J. Clin. Nutr. 2013, 97, 487-496. [CrossRef] [PubMed]

12. Chastin, S.F.M.; Buck, C.; Freiberger, E.; Murphy, M.; Brug, J.; Cardon, G.; O’Donoghue, G.; Pigeot, I.; Oppert, J.-M. Systematic literature review of determinants of sedentary behaviour in older adults: A DEDIPAC study. Int. J. Behav. Nutr. Phys. Act. 2015, 12, 127. [CrossRef] [PubMed]

13. World Health Organization. World Health Statistics 2014; World Health Organization: Geneva, Switzerland, 2014.

14. Kowal, P.; Chatterji, S.; Naidoo, N.; Biritwum, R.; Fan, W.; Lopez Ridaura, R.; Maximova, T.; Arokiasamy, P.; Phaswana-Mafuya, N.; Williams, S.; et al. Data resource profile: The World Health Organization Study on global AGEing and adult health (SAGE). Int. J. Epidemiol. 2012, 41, 1639-1649. [CrossRef] [PubMed]

15. World Bank. World Bank Analytical Classifications [Excel Spreadsheet]. Available online: Siteresources. worldbank.org/DATASTATISTICS/Resources/OGHIST.xls (accessed on 23 February 2018).

16. Naidoo, N. WHO Study on Global AGEing and Adult Health (SAGE) Waves 0 and 1-Sampling Information for China, Ghana, India, Mexico, Russia and South Africa; SAGE Working Paper No. 5; WHO: Geneva, Switzerland, 2012.

17. Process of Translation and Adaptation of Instruments. Available online: http://www.who.int/substance abuse/research_tools/translation/en/ (accessed on 1 May 2018).

18. World Health Organisation. WHO Study on global AGEing and adult health (SAGE). Available online: http:/ / www.who.int/healthinfo/sage/en/ (accessed on 1 May 2018).

19. Owen, N.; Sugiyama, T.; Eakin, E.E.; Gardiner, P.A.; Tremblay, M.S.; Sallis, J.F. Adults' sedentary behavior: Determinants and interventions. Am. J. Prev. Med. 2011, 41, 189-196. [CrossRef] [PubMed]

20. Howe, L.D.; Galobardes, B.; Matijasevich, A.; Gordon, D.; Johnston, D.; Onwujekwe, O.; Patel, R.; Webb, E.A.; Lawlor, D.A.; Hargreaves, J.R. Measuring socio-economic position for epidemiological studies in low- and middle-income countries: A methods of measurement in epidemiology paper. Int. J. Epidemiol. 2012, 41, 871-886. [CrossRef] [PubMed]

21. WHO Expert Consultation. Appropriate body-mass index for Asian populations and its implications for policy and intervention strategies. Lancet 2004, 363, 157-163. [CrossRef]

22. WHO Consultation on Obesity. Obesity: Preventing and Managing the Global Epidemic: Report of a WHO Consultation; WHO Technical Report Series: 894; WHO: Geneva, Switzerland, 2000.

23. World Health Organization (WHO). WHO Expert Committee on Physical Status: The Use and Interpretation of Anthropometry. In Physical Status: The use and Interpretation of Anthropometry: Report of a WHO Expert Committee; WHO Technical Report Series: 894; WHO: Geneva, Switzerland, 1995.

24. Cummings, S.R.; Studenski, S.; Ferrucci, L. A diagnosis of dismobility-Giving mobility clinical visibility: A mobility working group recommendation. JAMA 2014, 311, 2061-2062. [CrossRef] [PubMed]

25. World Health Organization. Consultation on Development of Standards for Characterization of Vision Loss and Visual Functioning; WHO: Geneva, Switzerland, 2003.

26. Maj, M.; D’Elia, L.; Satz, P.; Janssen, R.; Zaudig, M.; Uchiyama, C.; Starace, F.; Galderisi, S.; Chervinsky, A. Evaluation of two new neuropsychological tests designed to minimize cultural bias in the assessment of HIV-1 seropositive persons: A WHO study. Arch. Clin. Neuropsychol. 1993, 8, 123-135. [CrossRef] [PubMed]

27. Spreen, O.; Strauss, E. A Compendium of Neuropsychological Tests: Administration, Norms, and Commentary, 2nd ed.; Oxford University Press: New York, NY, USA, 1998.

28. Üstün, T.B.; Chatterji, S.; Kostanjsek, N.; Rehm, J.; Kennedy, C.; Epping-Jordan, J.; Saxena, S.; Korff, M.V.; Pull, C. Developing the World Health Organization Disability Assessment Schedule 2.0. Bull. World Health Organ. 2010, 88, 815-823. [CrossRef] [PubMed]

29. Üstün, T.B.; Kostanjsek, N.; Chatterji, S.; Rehm, J. Measuring Health and Disability: Manual for WHO Disability Assessment Schedule (WHODAS 2.0); WHO: Geneva, Switzerland, 2010.

30. Gaskin, C.J.; Lambert, S.D.; Bowe, S.J.; Orellana, L. Why sample selection matters in exploratory factor analysis: Implications for the 12-item World Health Organization Disability Assessment Schedule 2.0. BMC Med. Res. Methodol. 2017, 17, 40. [CrossRef] [PubMed]

31. Schmidt, S.; Mühlan, H.; Power, M. The EUROHIS-QOL 8-item index: Psychometric results of a cross-cultural field study. Eur. J. Public Health 2005, 16, 420-428. [CrossRef] [PubMed]

32. Armstrong, T.; Bull, F. Development of the world health organization global physical activity questionnaire (GPAQ). J. Public Health 2006, 14, 66-70. [CrossRef] 
33. Bull, F.C.; Maslin, T.S.; Armstrong, T. Global Physical Activity Questionnaire (GPAQ): Nine country reliability and validity study. J. Phys. Act. Health 2009, 6, 790-804. [CrossRef] [PubMed]

34. Herrmann, S.D.; Heumann, K.J.; Der Ananian, C.A.; Ainsworth, B.E. Validity and reliability of the Global Physical Activity Questionnaire (GPAQ). Meas. Phys. Educ. Exerc. Sci. 2013, 17, 221-235. [CrossRef]

35. Chu, A.H.Y.; Ng, S.H.X.; Koh, D.; Müller-Riemenschneider, F. Reliability and validity of the self- and interviewer-administered versions of the Global Physical Activity Questionnaire (GPAQ). PLoS ONE 2015, 10, e0136944. [CrossRef] [PubMed]

36. World Health Organization. Global Recommendations on Physical Activity for Health; WHO: Geneva, Switzerland, 2010.

37. Ekelund, U.; Steene-Johannessen, J.; Brown, W.J.; Fagerland, M.W.; Owen, N.; Powell, K.E.; Bauman, A.; Lee, I.M. Does physical activity attenuate, or even eliminate, the detrimental association of sitting time with mortality? A harmonised meta-analysis of data from more than 1 million men and women. Lancet 2016, 388, 1302-1310. [CrossRef]

38. Solomon, E.; Rees, T.; Ukoumunne, O.C.; Metcalf, B.; Hillsdon, M. Personal, social, and environmental correlates of physical activity in adults living in rural south-west England: A cross-sectional analysis. Int. J. Behav. Nutr. Phys. Act. 2013, 10, 129. [CrossRef] [PubMed]

39. Sallis, J.F. Age-related decline in physical activity: A synthesis of human and animal studies. Med. Sci. Sports. Exerc. 2000, 32, 1598-1600. [CrossRef] [PubMed]

40. Ingram, D.K. Age-related decline in physical activity: Generalization to nonhumans. Med. Sci. Sports Exerc. 2000, 32, 1623-1629. [CrossRef] [PubMed]

41. Eisenmann, J.C.; Wickel, E.E. The biological basis of physical activity in children: Revisited. Pediatr. Exerc. Sci. 2009, 21, 257-272. [CrossRef] [PubMed]

42. Bombak, A.E. Self-rated health and public health: A critical perspective. Front. Public Health 2013, 1, 15. [CrossRef] [PubMed]

43. Tak, E.; Kuiper, R.; Chorus, A.; Hopman-Rock, M. Prevention of onset and progression of basic ADL disability by physical activity in community dwelling older adults: A meta-analysis. Ageing Res. Rev. 2013, 12, 329-338. [CrossRef] [PubMed]

44. Cooper, A.J.M.; Lamb, M.J.E.; Sharp, S.J.; Simmons, R.K.; Griffin, S.J. Bidirectional association between physical activity and muscular strength in older adults: Results from the UK Biobank study. Int. J. Epidemiol. 2017, 46, 141-148. [CrossRef] [PubMed]

45. Lindsay Smith, G.; Banting, L.; Eime, R.; O'Sullivan, G.; van Uffelen, J.G.Z. The association between social support and physical activity in older adults: A systematic review. Int. J. Behav. Nutr. Phys. Act. 2017, 14. [CrossRef] [PubMed]

46. Sun, F.; Norman, I.J.; While, A.E. Physical activity in older people: A systematic review. BMC Public Health 2013, 13, 449. [CrossRef] [PubMed]

47. Shephard, R.J. Limits to the measurement of habitual physical activity by questionnaires. Br. J. Sports Med. 2003, 37, 197-206. [CrossRef] [PubMed]

48. Sallis, J.F.; Saelens, B.E. Assessment of physical activity by self-report: Status, limitations, and future directions. Res. Q. Exerc. Sport 2000, 71, 1-14. [CrossRef] [PubMed]

49. Heesch, K.C.; van Uffelen, J.G.Z.; Hill, R.L.; Brown, W.J. What do IPAQ questions mean to older adults? Lessons from cognitive interviews. Int. J. Behav. Nutr. Phys. Act. 2010, 7, 35. [CrossRef] [PubMed]

50. Guthold, R.; Louazani, S.A.; Riley, L.M.; Cowan, M.J.; Bovet, P.; Damasceno, A.; Sambo, B.H.; Tesfaye, F.; Armstrong, T.P. Physical activity in 22 African countries. Am. J. Prev. Med. 2011, 41, 52-60. [CrossRef] [PubMed]

(C) 2018 by the authors. Licensee MDPI, Basel, Switzerland. This article is an open access article distributed under the terms and conditions of the Creative Commons Attribution (CC BY) license (http:/ / creativecommons.org/licenses/by/4.0/). 